\title{
The Vanishing and Appearing Sources during a Century of Observations Project. I. USNO Objects Missing in Modern Sky Surveys and Follow-up Observations of a "Missing Star"
}

\author{
Beatriz Villarroel ${ }^{1,2}$, Johan Soodla ${ }^{3}$, Sébastien Comerón $^{4}$, Lars Mattsson ${ }^{1}$, Kristiaan Pelckmans ${ }^{3}$, Martín López-Corredoira ${ }^{2,5}$ (i), \\ Kevin Krisciunas $^{6}$ (10), Eduardo Guerras ${ }^{7}$ (10), Oleg Kochukhov ${ }^{8}$ (10), Josefine Bergstedt ${ }^{8}$, Bart Buelens ${ }^{9,10}$, Rudolf E. Bär ${ }^{11}$, \\ Rubén Cubo $^{3}$, J. Emilio Enriquez ${ }^{12}$ (1), Alok C. Gupta ${ }^{13}$ (1) Iñigo Imaz $^{8}$, Torgny Karlsson ${ }^{14}$, M. Almudena Prieto ${ }^{2,5}$, \\ Aleksey A. Shlyapnikov ${ }^{15}$, Rafael S. de Souza ${ }^{16}$, Irina B. Vavilova ${ }^{17}$, and Martin J. Ward ${ }^{18}$ \\ ${ }^{1}$ Nordita, KTH Royal Institute of Technology and Stockholm University, Roslagstullsbacken 23, SE-106 91 Stockholm, Sweden; beatriz.villarroel@ su.se \\ ${ }^{2}$ Instituto de Astrofisica de Canarias, Avda Via Lactea S/N, La Laguna, E-38205, Tenerife, Spain \\ ${ }^{3}$ Department of Information Technology, Uppsala University, Uppsala, Sweden \\ ${ }^{4}$ University of Oulu, Astronomy Research Unit, FI-90014 Oulu, Finland \\ 5 Departamento de Astrofisica, Universidad de La Laguna, E-38206 La Laguna, Tenerife, Spain \\ ${ }^{6}$ Department of Physics and Astronomy, Texas A\&M University, 4242 TAMU, College Station, TX 77843, USA \\ ${ }^{7}$ Homer L. Dodge Department of Physics and Astronomy, The University of Oklahoma, Norman, OK 73019, USA \\ ${ }_{9}^{8}$ Department of Physics and Astronomy, Uppsala University, Uppsala, Sweden \\ ${ }^{9}$ Statistics Netherlands, Methodology Department, Heerlen, The Netherlands \\ ${ }^{10}$ VITO, Diepenbeek Genk, Flanders, Belgium \\ ${ }^{11}$ Institute for Particle Physics and Astrophysics, ETH Zurich, Wolfgang-Pauli-Strasse 27, CH-8093 Zurich, Switzerland \\ ${ }^{12}$ Department of Astronomy, University of California Berkeley, 501 Campbell Hall \#311, Berkeley, CA 94720, USA \\ ${ }^{13}$ Aryabhatta Research Institute of Observational Sciences (ARIES), Manora Peak, Nainital, 263 002, India \\ ${ }^{14}$ Department of Immunology, Uppsala University, Uppsala, Sweden \\ ${ }^{15}$ Stellar Physics Department, Crimean ${ }^{19}$ Astrophysical Observatory, 298409, Nauchnyj, Crimea \\ ${ }^{16}$ Department of Physics \& Astronomy, University of North Carolina at Chapel Hill, NC 27599-3255, USA \\ ${ }^{17}$ Main Astronomical Observatory of the NAS of Ukraine, 27, Akademik Zabolotny St., Kyiv, 03143, Ukraine \\ ${ }^{18}$ Centre for Extragalactic Astronomy, Department of Physics, Durham University, South Road, Durham DH1 3LE, UK \\ Received 2019 July 25; revised 2019 November 6; accepted 2019 November 11; published 2019 December 12
}

\begin{abstract}
In this paper we report the current status of a new research program. The primary goal of the "Vanishing and Appearing Sources during a Century of Observations" project is to search for vanishing and appearing sources using existing survey data to find examples of exceptional astrophysical transients. The implications of finding such objects extend from traditional astrophysics fields to the more exotic searches for evidence of technologically advanced civilizations. In this first paper we present new, deeper observations of the tentative candidate discovered by Villarroel et al. in 2016. We then perform the first searches for vanishing objects throughout the sky by comparing 600 million objects from the US Naval Observatory Catalogue (USNO) B1.0 down to a limiting magnitude of $\sim 20-21$ with the recent Pan-STARRS Data Release-1 (DR1) with a limiting magnitude of $\sim 23.4$. We find about 150,000 preliminary candidates that do not have any Pan-STARRS counterpart within a $30^{\prime \prime}$ radius. We show that these objects are redder and have larger proper motions than typical USNO objects. We visually examine the images for a subset of about 24,000 candidates, superseding the 2016 study with a sample 10 times larger. We find about 100 point sources visible in only one epoch in the red band of the USNO, which may be of interest in searches for strong M-dwarf flares, high-redshift supernovae, or other categories of unidentified red transients.
\end{abstract}

Unified Astronomy Thesaurus concepts: Astrobiology (74); Photographic photometry (1229); Surveys (1671); Transient sources (1851); Gamma-ray transient sources (1853)

\section{Introduction}

Many of the hottest topics in current astronomical research concern the physics of extreme transient phenomena, such as gravitational wave events, gamma-ray bursts, fast radio bursts (FRBs) or outbursts of active galactic nuclei (AGNs). Although we are gaining a better understanding of the physical processes governing them, our understanding of transient phenomena in general is inevitably limited by the a priori assumptions that go into the data collection when we design our observations. With the advent of the Virtual Observatory in the early 2000s, astronomers suggested that very large surveys together with

\footnotetext{
${ }^{19}$ While the AAS journals adhere to and respect UN resolutions regarding the designations of territories (available at http://www.un.org/press/en), it is our policy to use the affiliations provided by our authors on published articles.
}

state-of-the-art developments in information technology could efficiently be used to probe rare or unusual astrophysical phenomena by expanding the parameter space beyond our current knowledge (Djorgovski 2000; Djorgovski et al. 2001). An example of such a rare class of object that would not have been discovered unless specifically looked for is that of Hippke's star. This emerged from a search for artificially modified pulsations in Cepheid variables and led to the discovery of rare objects with two regimes with double pulsation periods of both long and short duration (Hippke et al. 2015).

Another example of objects that may be missed in transient surveys, unless specifically looked for, are the rare failed supernovae (Kochanek et al. 2008), which occur when a star collapses almost directly to form a black hole. Recently, the 
possible detection of a failed supernova in a nearby galaxy has been reported (Adams et al. 2017a, 2017b). The more exotic the phenomenon, the more likely we are to miss it in the observational data due to our preconceptions and the duration and frequency of the sampling.

In this paper we describe the "Vanishing and Appearing Sources during a Century of Observations" (VASCO) project, ${ }^{20}$ a multitask effort aimed at finding some of the most unusual variable phenomena and other astrophysical anomalies based on existing sky surveys. We also aim to develop a citizen science branch of VASCO, and indeed the basic philosophy behind the project was first described for a wider audience by Mattsson \& Villarroel (2017).

VASCO is primarily centered around searches for vanishing objects observed in the sky and beyond the Earth's local environment. Unless a star collapses directly into a black hole, there is no known physical process by which it could physically vanish. If such examples exist this makes it interesting for searches for new exotic phenomena or even signs of technologically advanced civilizations (Villarroel et al. 2016). Vanishing stellar events currently are missed and hence go undetected in most ongoing all-sky surveys. Villarroel et al. (2016) found only one such tentative candidate after a crossmatch between 10 million USNO sources and the Sloan Digital Sky Survey (SDSS; York et al. 2000). Even if we discover a star that appears to vanish, it is an observational challenge to determine whether the object really vanished or just faded below the detection limit.

The VASCO project aims to find both vanishing and appearing sources as well as objects that show extreme variability on extended timescales (many decades), by comparing sky scans that are nearly a century old $(\sim 70 \mathrm{yr})$ with modern-day astronomical surveys. Compared to recent transient facilities such as the Zwicky Transient Facility (ZTF), which commenced operations in 2018, we are probing a significantly longer time window-about $70 \mathrm{yr}$ - by investigating events that occurred between the epoch of the US Naval Observatory Catalogue (USNO) (Monet et al. 2003) and the recent Pan-STARRS survey that has multiple detections for each astronomical source (Kaiser et al. 2002). Prior efforts to probe these long timescales have been led by the "Digital Access to a Sky Century @ Harvard" (DASCH) project (Grindlay et al. 2012), which has digitized more than 450,000 plates with a full-sky coverage. The plates used were taken during the years 1890-1990 and had a limiting magnitude of $B \sim 14$ (or $V \sim 15$ ). Among the modern CCD surveys the Catalina Real-Time Transient Survey (CRTS) has the longest time span $(\sim 14 \mathrm{yr})$, with a total sky coverage of about 30,000 deg ${ }^{2}$ and about 500 million light curves (Drake et al. 2009; Mahabal et al. 2011; Djorgovski et al. 2012). The data, which are public, extend to $V \sim 19-21 \mathrm{mag}$ per exposure and are based on CCD photometry taken at a large number of epochs. The CRTS has so far discovered about 17,000 optical transients, among them many superluminous and peculiar supernovae, about 1500 cataclysmic variables, and about 4000 variable AGNs.

Using a relatively long time window of $\sim 70 \mathrm{yr}$, in combination with a large sample size, increases the probability of finding extremely rare events. Clearly this is still a very short time duration from a cosmological perspective, but it nevertheless sets an upper limit on the incidence of vanishing or

\footnotetext{
${ }^{20}$ https://vasconsite.wordpress.com
}

appearing sources. In addition there are a number of recently discovered astronomical transients that occur over significantly longer timescales than common variable stars, which vary on periods from weeks to a few years. For example, hypervariable AGNs (Lawrence et al. 2016; Kankare et al. 2017) were discovered by comparing two astronomical surveys 10 years apart. More than $95 \%$ of extragalactic objects exhibiting this long-term variability show the presence of an AGN (Drake et al. 2019). Hypervariable AGNs exhibit still poorly understood long-term variability that could have various causes, e.g., microlensing events, superluminous supernovae in the accretion disk (Graham et al. 2017), or changes in their Eddington ratio (Graham et al. 2019). These hypervariable AGNs have been extensively studied with the CRTS.

The DASCH project has reported other interesting findings while probing these timescales. For example, it revealed longterm dimming of $\mathrm{K}$ giants (Tang et al. 2010), and resulted in the discovery of an unusual nova with an outburst (or flare) in 1942 that was followed by a $10 \mathrm{yr}$ decline (Tang et al. 2012). Peculiar transients have also been found in the CRTS, e.g., the very long-lasting Type IIn SN 2008iy, which took over 400 days to reach its peak brightness (Mahabal et al. 2009).

Our limiting magnitude is much deeper (Pan-STARRS: $r \sim 23.4)$ than DASCH $(V \sim 15)$ and we focus specifically on the most extreme events that appeared above, or disappeared below, the detection limit in searches for the most extreme astronomical events and objects. Our time span is significantly longer than that of the CRTS survey. One may expect to find R Coronae Borealis ( $\mathrm{R}$ CrB) stars. These are carbon-rich supergiant stars that can dim up to 9 mag with irregular time intervals, where the fading happens on timescales ranging from a few months to years. These eruptive objects have a poorly understood origin, while the most prominent hypothesis is that they formed from mergers of two white dwarfs or are the result of $\mathrm{He}$ flashes in the central star of a planetary nebula (Clayton 2012). Today we know of $\sim 150 \mathrm{R} \mathrm{CrB}$ stars in our Galaxy (Tisserand et al. 2018) and expect about 5000 to exist.

Highly variable objects such as eclipsing binaries, Cepheids, RR Lyrae, R Coronae Borealis, dwarf novae, and highly variable AGNs are expected to be detected by VASCO, as their luminosity falls below or rises above the Pan-STARRS detection limit of $r \sim 23.4$. As the limiting magnitude of USNO is around $\sim 20-21$, this corresponds to a change of at least 2 mag during the time period of $70 \mathrm{yr}$. Mira variables may vary up to 10 mag on timescales of a few years. Objects similar to these variables may eventually be rediscovered during follow-up observations with larger telescopes or by patiently waiting for the object to reappear a few months or many years later.

Moreover, VASCO may also discover objects that are only visible in one epoch and then disappear in later surveys. Nearby stars with high proper motion will fall into this category. Outbursts in AGNs caused by relativistic jet activity or major increases in accretion will also give short-term signatures in the optical that fade away in a few months or years, e.g., Prieto (1997) or Mack et al. (2009). Also, transients such as supernovae and tidal disruption events can be detected in this way. But natural astrophysical sources are not the only possible sources to discover. Modern programs to search for extraterrestrial intelligence are nowadays preparing and executing searches for interstellar optical laser communication, especially in the red and infrared. Therefore, it is of great interest to 


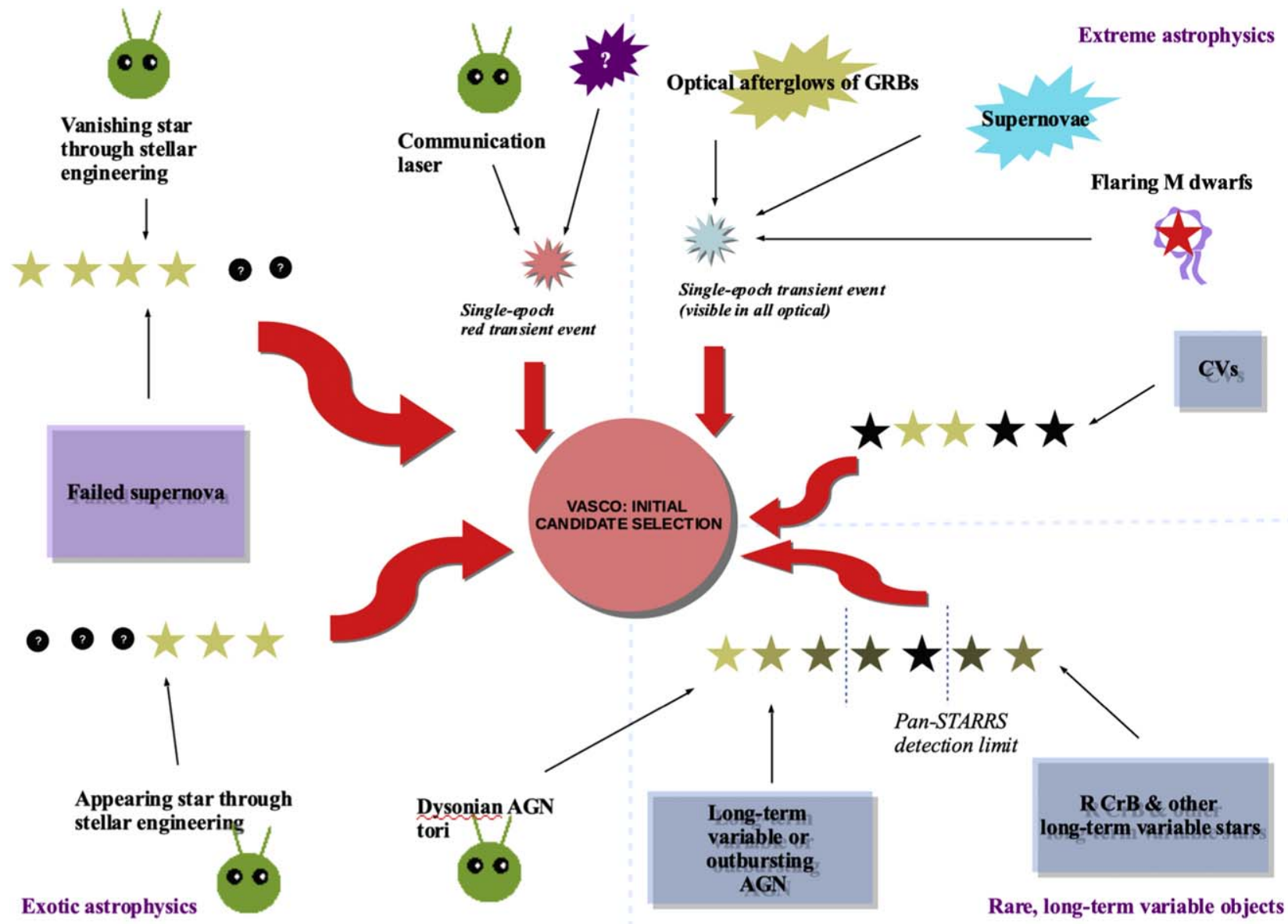

Figure 1. VASCO candidate selection. Once instrumental flaws and errors are removed, we expect different types of objects to be included in the VASCO "mismatch" sample. A particular focus is given to USNO objects that have several detections before vanishing or to objects that are brighter than 18.4 mag in USNO and thus have dimmed at least $5 \mathrm{mag}$. Rare, long-term variable objects may seem to appear or disappear in the USNO and Pan-STARRS catalogs as they rise above or fall below the detection limit. Among the daily but extreme astrophysical phenomena, we may detect some fast transients only seen at one epoch. Fast transients only seen in the red image could be the result of strongly redshifted transients or less well-known physical phenomena, or also a result of interstellar communication with red, monochromatic lasers. The VASCO time baseline that probes variability over several decades provides opportunities to study multiple phenomena.

identify any transient that is only visible once, provided that we can later exclude those events that may be a result of plate defects, cosmic rays, and other detection flaws. Figure 1 shows what kind of objects we may collect in our first candidate selection. In order to pinpoint the nature of each candidate, one must reconstruct its light curve, which can be done with help of old and modern archives and by making deeper observations.

In this paper, we start by examining the tentative candidate reported by Villarroel et al. (2016). We present the results of indepth archival searches, and also some new observations of this object. After examining the candidate, we cross-match the USNO and Pan-STARRS surveys. The current USNO sample is increased by a factor of 60 in comparison to the sample used by Villarroel et al. (2016), because we use about $60 \%$ of the USNO catalog for the cross-matching. In contrast to the previous work, we also include objects with non-zero proper motion. In Section 3 we discuss the properties of the "Mismatch Sample." We conduct a preliminary analysis of the images in the "SDSS subsample," which includes about $15 \%$ of the "Mismatch Sample." The preliminary list of candidates that resulted from visual examination has been studied at seven epochs (five POSS surveys, SDSS, and PanSTARRS). While this endeavor may include many objects similar to those that time-dependent surveys such as the CRTS and ZTF already detect, we particularly emphasize single-time transients with large amplitudes $\Delta m>5$ mag and objects that have been observed in more than one image prior to "disappearance" in order to collect the most exotic and extreme phenomena. Finally, we detail the general design and methodology of the VASCO project, as it is currently planned to be carried out over the coming years, including a citizen science project.

In a separate paper (K. Pelckmans et al. 2019, in preparation) we propose a machine-learning-based tool aimed to facilitate the planned citizen science project.

\section{The "Vanishing" Star in Villarroel et al. (2016)}

Villarroel et al. (2016) identified a candidate, but the candidate was not robust enough to make a convincing case for an example of a vanishing star. In the USNO catalog this object was listed as having two detections: one was clearly visible and point-like in the POSS-I red-band image and the other was less clearly visible in the POSS-II red band. We decided to re-examine it, both by reassessing the old observations and by following up with some new imaging obtained with larger telescopes. 
Table 1

Summary of the Observations

\begin{tabular}{|c|c|c|c|}
\hline Archive/telescope & Detection? & Detection limit (mag) & Date of observation \\
\hline POSS-I O blue $\sim 4100 \AA$ (Palomar) & no & 21 & 1950 Mar 16 \\
\hline POSS-I E red $\sim 6500 \AA$ (Palomar) & yes, $19.7 \mathrm{mag}$ & 20 & 1950 Mar 16 \\
\hline POSS-II J blue $\sim 4400 \AA$ (Palomar) & no & 22.5 & 1993 May 19 \\
\hline POSS-II F red (Palomar) 6600 А (Palomar) & offset/dirt? & 20.8 & 1993 Mar 22 \\
\hline POSS-II N IR 8400 ^ (Palomar) & faint detection/noise? & 19.5 & 1993 Mar 4 \\
\hline Quick-V Northern & faint detection/noise? & $?$ & 1982 May 19 \\
\hline SDSS (New Mexico 2.5 m) & no & 23 & $?$ \\
\hline Pan-STARRS & no & 23.4 & $?$ \\
\hline Gaia & no & 21 & $?$ \\
\hline$G A L E X-5$ & no & $?$ & $?$ \\
\hline Ukraine $\mathrm{VO}^{\mathrm{a}, \mathrm{b}, \mathrm{c}}$ & no & $\sim 17$ & 1982 May 26 \\
\hline Ukraine VO & no & $\sim 17$ & 1987 Apr 23 \\
\hline Ukraine VO & no & $\sim 17$ & 1991 May 11 \\
\hline Ukraine VO & no & $\sim 17$ & 1993 May 10 \\
\hline Ukraine VO & no & $\sim 17$ & 1993 May 11 \\
\hline WISE & counterpart $5 " ! 8$ north? & $?$ & $?$ \\
\hline CAMELOT (La Palma, $1.0 \mathrm{~m}$ ), red & something at noise level? & 24.7 & 2018 May 9 \\
\hline ALFOSC (NOT, $2.5 \mathrm{~m}$ ), red, obs. block 1 & $24.26 \pm 0.02$ mag (southern object) & $\sim 25.5-26$ & 2018 May 16 \\
\hline ALFOSC (NOT, $2.5 \mathrm{~m}$ ), red, obs. block 2 & $24.26 \pm 0.02 \mathrm{mag}$ (southern object) & $\sim 25.5-26$ & 2018 May 23 \\
\hline ALFOSC (NOT, $2.5 \mathrm{~m}$ ), red, obs. block 1 & $25.18 \pm 0.03 \mathrm{mag}$ (northern object) & $\sim 25.5-26$ & 2018 May 16 \\
\hline ALFOSC (NOT, $2.5 \mathrm{~m}$ ), red, obs. block 2 & $25.18 \pm 0.03 \mathrm{mag}$ (northern object) & $\sim 25.5-26$ & 2018 May 23 \\
\hline Magellan telescope (Baade $6.5 \mathrm{~m}$ ), blue & no & $?$ & 2018 Jun 5 \\
\hline
\end{tabular}

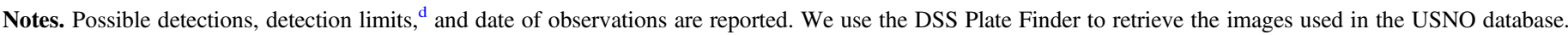

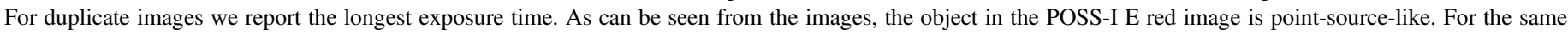

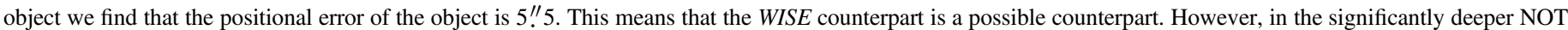
images we find both the WISE counterpart and two possible candidates very close to the position of the original USNO object.

${ }^{\text {a }}$ Ukraine VO archives are described by Vavilova et al. (2012) and Vavilova (2016).

${ }^{\mathrm{b}}$ http://gua.db.ukr-vo.org/archivespecial.php

${ }^{c}$ http://ukr-vo.org/digarchives/index.php?b5\&1

${ }^{\mathrm{d}}$ Limiting magnitudes for POSS are taken from Djorgovski et al. (1998).

\subsection{Observations with CAMELOT at IAC80}

We observed with the IAC80 telescope, which is a part of the Teide Observatory and belongs to the Instituto de Astrofisica de Canarias (IAC), located on the island of Tenerife (Spain). We used the CAMELOT (Camara Mejorada Ligera del Observatorio del Teide") instrument in service mode and obtained nine exposures of 30 minutes each in the red filter. The pixel size is 0" 304 and the limiting magnitude about 24.7 in the Sloan $r$-band.

\subsection{Observations with ALFOSC at NOT}

We made even deeper observations (down to $r \sim 25.5-26$ ) with the help of the Alhambra Faint Object Spectrograph and Camera (ALFOSC) instrument at the Nordic Optical Telescope (NOT, La Palma, Spain) in service mode and fast-track observations. The goal was to carry out deep enough observations to be able to detect a point source at the 25 th magnitude level with a signal-to-noise ratio of at least 9 or 10 , using deep Gunn $r^{\prime}$-band imaging. Assuming an airmass of 1.5, seeing of $1^{\prime \prime}$, and a gray night, we estimated that about four hours of observation time were needed. Six exposures of $900 \mathrm{~s}$ were taken. For the resulting images, the pixel size was 0.'214 and the limiting magnitude about $25.5-26.0$ in the $r$-filter.

\subsection{Results from the Observations}

We summarize what we know about the existing observations of the object so far in Table 1. The table presents old archival observations as well as new ones we have performed during the follow-up.

We first examine the old POSS images. As we can see from the table, the minimum requirement of two detections (on which USNO is based) is not clear for this particular object. Only one strong confirmation (POSS-I E plate) exists. Unlike an artifact, the object appears to be point-source-like in the POSS-I E plate. See Figure 2. One possibility is that this object is a star with significant proper motion and it moved entirely out of the image.

We compare the POSS-I E image with the new images taken with the NOT. See Figure 3. In the NOT imagery we find two objects very close to the original USNO location. One of the objects is located 2."4 southwest of the USNO object, and the second is 1 ". 4 northwest of the USNO object. However, the resolution in the POSS-I E band is about 1".7 per pixel, and the displacement of the two reported objects is therefore within the error, in particular for the closer object only 1". 4 away.

The colors may give a clue. The original USNO object was only seen in the red band. While the nearby counterpart from the Wide-field Infrared Survey Explorer (WISE) is seen both in the blue band with the Magellan telescope and in the red band with NOT, the NOT objects can only be seen in the red. This may support the hypothesis that the object from the 1950s and one of the objects seen in NOT are likely to be the same. But if so, the brighter (southwest) object has dropped about 4.2-4.3 mag in the $r$-band and also moved a bit.

One may wonder what is the probability of observing a new, unrelated object with NOT within 2."5 of the stated position in 


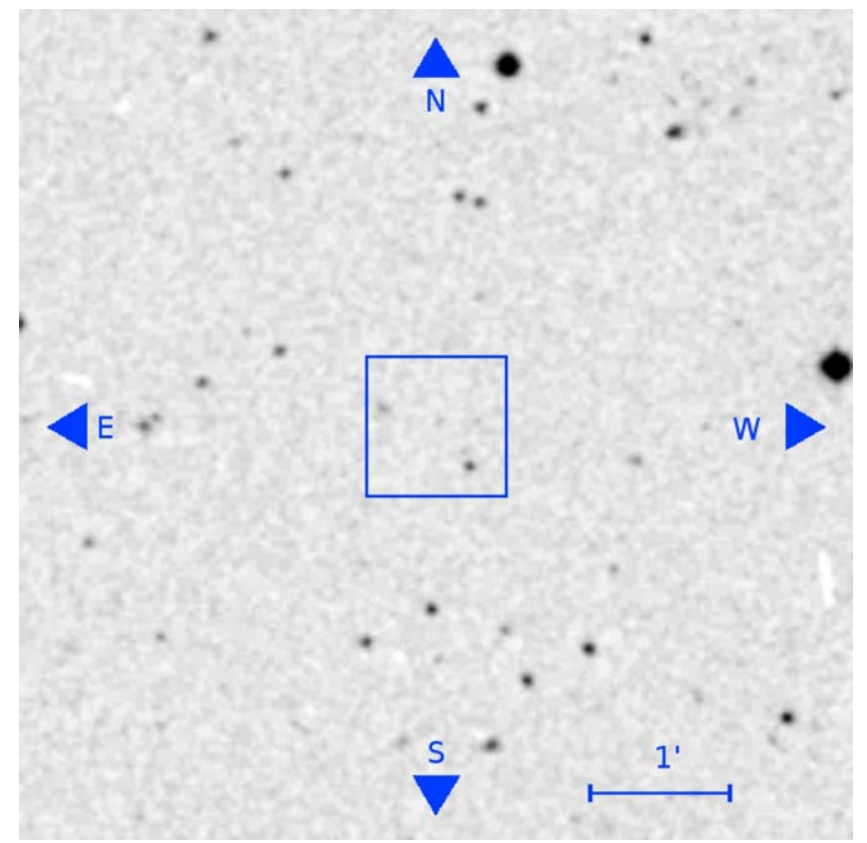

Figure 2. The POSS-I E red observation of the object in Villarroel et al. (2016), which is centered on the small square.

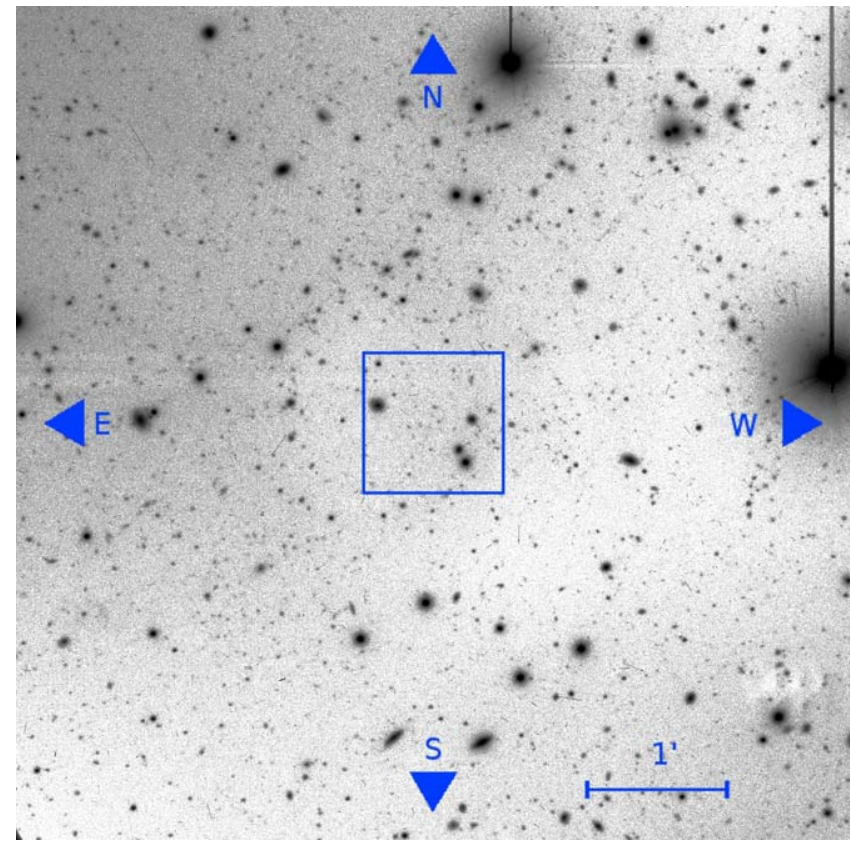

Figure 3. The NOT observation of the object in Villarroel et al. (2016). The image was constructed by summing two observing blocks taken on 2018 May 16 and 23. There are two potential counterparts 1 ."4 northwest and 2.4 southwest of the USNO object's original position.

USNO, if going $4.2 \mathrm{mag}$ deeper. However, probability estimates of this sort are of little help when we search deliberately for outliers in big data sets covering billions of objects.

\subsection{Could It Have Moved?}

One possibility is that our target is a star with a fairly high proper motion (despite being cataloged in USNO as having no proper motion), and that it has moved substantially from its original position. If we compare the two different red plates from the POSS within a reasonable angular distance, we should be able to find the missing object by seeing an appearing object in the later POSS image from 1993.

Assuming a maximum proper motion of 6" per year (slightly larger offset than the positional error of 5."5), we know that between 1950 and 1993 the star will not have moved more than $4 ! 3$ in any direction during these years. We therefore extract red filter images from POSS-I (from 1950) and POSS-II (1993) with a field of view corresponding to $9^{\prime} \times 9^{\prime}$. We inspect these visually by "blinking" them. The few objects that "appear" in the later epoch turn out to also exist in the SDSS images, which means they simply were not resolved in the previous epoch. No other "appearing" objects could be seen in the later epoch, which means that we can quite safely reject the hypothesis of a fast moving star. Solar system objects typically are bluer (as they shine by reflected sunlight), although exceptions of course exist. From the DSS Plate Finder ${ }^{21}$ we see that the POSS-I E red and POSS-I O blue images were taken about half an hour apart, but nothing is visible in the blue image at the position of the star. Given the exposure time of 45 minutes of the POSS-I E red image, if our object were an asteroid that quickly moved out of the field, it would have left a stripe (and not be point-like).

\subsection{Was It Possibly an Image Defect?}

The final hypothesis that could rule out the idea of a transient or variable event in the 1950s plate is the simplest explanation of all: defects in the old photographic plates from POSS-I. While the USNO-B1.0 should be cleaned up from a fair number of these artifacts, and a separate list by Barron et al. (2008) could have included our target but did not, our target still has survived thanks to the two detections listed in USNO.

We reanalyze the POSS images based on high-resolution data from STScI Digitized Sky Survey (see footnote 20). We see several things: only the detection from the POSS-I E red plate taken on 1950 March 16 is secure. The second detection - which we believe is based on the POSS-II F image from 1993 March 22-is slightly offset and possibly not the same object (even if listed as the same; here the low resolution may have played a role). Of all the other images available on that server covering that particular sky region-Quick-V Northern (1982), Poss-I O (blue, 1950), POSS-II Blue (1986), POSS-II N (1993), POSS-II N (1996)—none of them convincingly shows the object. Some hints of an object may be seen at the given position in the Quick-V Northern image from 1982, but not in a way that would allow us to confirm the detection quantitatively because the signal-to-noise ratio is very low.

While plate defects in USNO very seldom are star-like (Madsen \& Gaensler 2013), some of the star-like sources could in principle be photographic plate defects. These defects can be created when a small dust particle sticks to the plate during the exposure, or when microspots form after years of storage. Greiner et al. (1990) proposed examining original plates with a microscope in reflected light to help sort out which events we see are real astronomical events and which are pure plate defects. The way to be sure when dealing with old photographic plate material is to investigate the photographic plates themselves under a microscope.

\footnotetext{
${ }^{21}$ https://archive.stsci.edu/cgi-bin/dss_plate_finder
} 
Unfortunately, we do not have access to the original plates. However, by comparing the point-spread function (PSF) of the object to the PSF of typical stars in the same field, one can see whether the object is likely to be a plate flaw or a real star. See Section 4.2. If an object has a considerably smaller PSF than a real star as measured on the given photographic plate, it may be discarded as a plate flaw. Our object appears to be like many astronomical point sources and has a PSF comparable to the real stars on the plate. This suggests that it is not a plate defect.

\section{New Searches, New Samples}

We continue our exploratory journey by cross-matching the USNO and Pan-STARRS catalogs in searches for better candidates.

\subsection{Starting Samples}

The USNO-B1.0 catalog (Monet et al. 2003) presents the best old sky survey we can use in the optical because it goes deep enough $(r \sim 20)$ and contains one billion astronomical objects. It has all-sky coverage. This allows us to find astronomical transients that occurred before the birth of the all-sky transient surveys. Each object is supposedly detected at least twice in two widely separated epochs in the Palomar Sky Survey (POSS). ${ }^{22}$ The data were obtained in one blue band and one red band, and for some objects, also in the infrared. The Pan-STARRS catalog has about 2-3 billion objects and is at present the largest digital sky survey, with observations started in 2010. It covers the entire sky down to declinations decl. $\sim$ $-30^{\circ}$. Information about the Pan-STARRS data products is described in a series of articles (Chambers et al. 2016; Flewelling et al. 2016; Magnier et al. 2016a, 2016b, 2016c; Waters et al. 2016). Our PS1 data set is an offline version kindly provided by the Pan-STARRS collaboration.

One may wonder if it would not be better to directly search for vanishing or appearing objects only using internally consistent data sets such as Gaia or Pan-STARRS, containing about 2-3 billion objects each, where each object has photometry done multiple times during $5 \mathrm{yr}$ of observations. The CRTS has homogeneous data and time baselines up to $14 \mathrm{yr}$ with CCD data. However, when one compares the digitized sky from USNO plates with the sky from PanSTARRS, the significantly longer time span $(\sim 70 \mathrm{yr})$ changes the effective volume of the data set over which any event could have been observed. Extremely rare events are much more likely to be found in surveys that combine both a long time baseline and deep photometry. The DASCH survey may have $100 \mathrm{yr}$ of photometry, but it has a limiting magnitude around $V \sim 15$. The longer time span allows us to discover extreme variables with a characteristic timescale of several decades, longer than the typical five years of the intermediate Palomar Transient Factory (iPTF). An example of a vanishing-star event that is not expected to happen in the Milky Way more often than once every few hundred years is the hypothetical failed supernova event (see Appendix). The VASCO time baseline and depth in photometry make it possible to discover such events. Of course, we expect also to detect many objects that vary on shorter timescales.

\footnotetext{
22 POSS-I was carried out from 1950 to 1966, and POSS-II from 1987 to 1999 .
}

\subsection{Cross-matching the USNO and Pan-STARRS Catalogs}

The goal of the cross-matching algorithm used in this paper is to make a list of USNO objects that do not have a PanSTARRS counterpart within a certain distance threshold (e.g., $\left.30^{\prime \prime}\right)$. In the paper by Villarroel et al. (2016) the sizes of the starting samples used were, on average, about 10 million USNO objects. However, using the full catalogs of USNOB1.0 and Pan-STARRS DR1 is more of a practical challenge, because the databases we have make up about $1 \mathrm{~TB}$ in size (roughly $300 \mathrm{~GB}$ and $700 \mathrm{~GB}$ respectively). This creates a problem of efficiency in the cross-matching process, which could last unacceptably long if not done smartly.

We use a 3 TB cloud environment provided by the Uppsala Multidisciplinary Center for Advanced Computational Science (UPPMAX), which is part of the Swedish National Infrastructure for Computing (SNIC). The cross-matching is done in the environment of SQlite3, and carried out by parallellizing the cross-matching process by breaking down the USNO and Pan-STARRS databases into many smaller ones with the help of smart index methods. This enables the cross-matching process to be done effectively in smaller subsets than if using the whole databases. All the technical details of the crossmatching are described by Soodla (2019).

The cross-matching procedure in VASCO differs from a traditional cross-matching between two catalogs, because we are searching for missing objects rather than corresponding objects.

In a traditional cross-match, one uses an object from catalog A and tries to identify the same object in catalog B using the coordinates (and additional properties such as fluxes, surface densities of sources, etc.). Due to proper motion, variability, and many other factors, it can be quite challenging to verify whether the object within a certain radius in catalog $B$ is the same object. A typical cross-match radius in traditional projects is $3^{\prime \prime}-5^{\prime \prime}$. If one uses instead a large cross-matching radius (e.g., $\left.30^{\prime \prime}\right)$, there are often several possible matches, which means we may have a number of false positives among the cross-matches, and we have included spurious objects in the resulting catalog.

In our particular case, the cross-matching is not a traditional cross-match. When we take an object from catalog A and try to look for a "vanishing" object in catalog B, we only care to know that no object at all resides at the given position in the second catalog B. If one uses a small "cross-match" radius such as $5^{\prime \prime}$, this leads to a large number of mismatches because various astrometric issues enter, including the proper motion of objects. However, by extending the "cross-match" radius to 30 ", one implicitly takes care of issues related to proper motion, except possibly for nearby red dwarfs or white dwarfs. That would make sure that USNO objects with proper motions less than $0 . " 4 \mathrm{yr}^{-1}$ over a $70 \mathrm{yr}$ baseline are directly excluded from the resulting "mismatch" sample. The downside with this method is that one misses out on potential mismatches because false negatives enter the picture. This means that with a large cross-match radius our mismatches are very likely to be real ones, but we underestimate the number of mismatches (and hence we miss candidates). For objects with proper motions larger than $\sim 0$." $4 \mathrm{yr}^{-1}$, the displacement in coordinates is visible and easy to identify by blinking images. See Section 3.2.1.

From the USNO and Pan-STARRS J2000 coordinates we determine whether a counterpart exists or not within a certain angular distance. The USNO objects not having a counterpart 

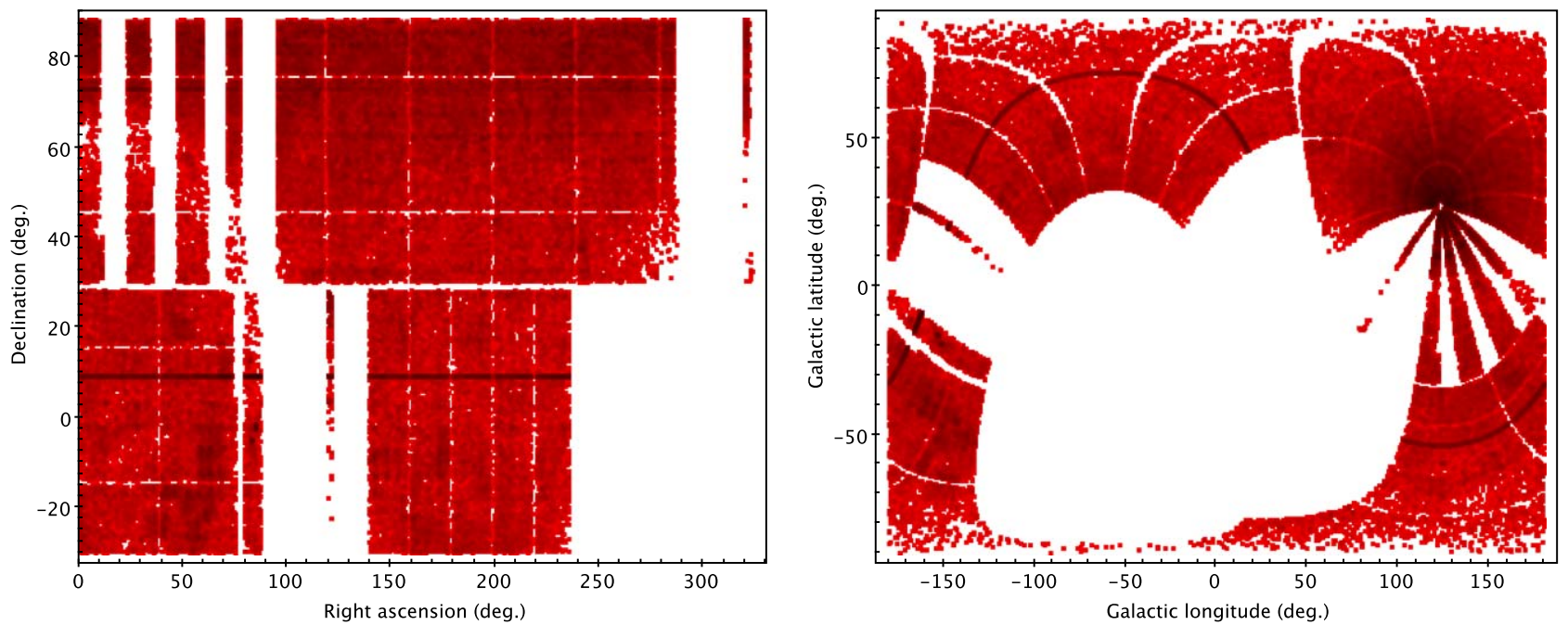

Figure 4. The distribution of the $\sim 150,000$ candidates on the sky. The left plot shows equatorial coordinates, and the right plot shows Galactic longitudes. Some regions are unsampled in the cross-match, as can be seen by the empty regions, in particular R.A. $\left(240^{\circ}, 360^{\circ}\right)$ and decl. $\left(-30^{\circ}, 30^{\circ}\right)$ in equatorial coordinates.

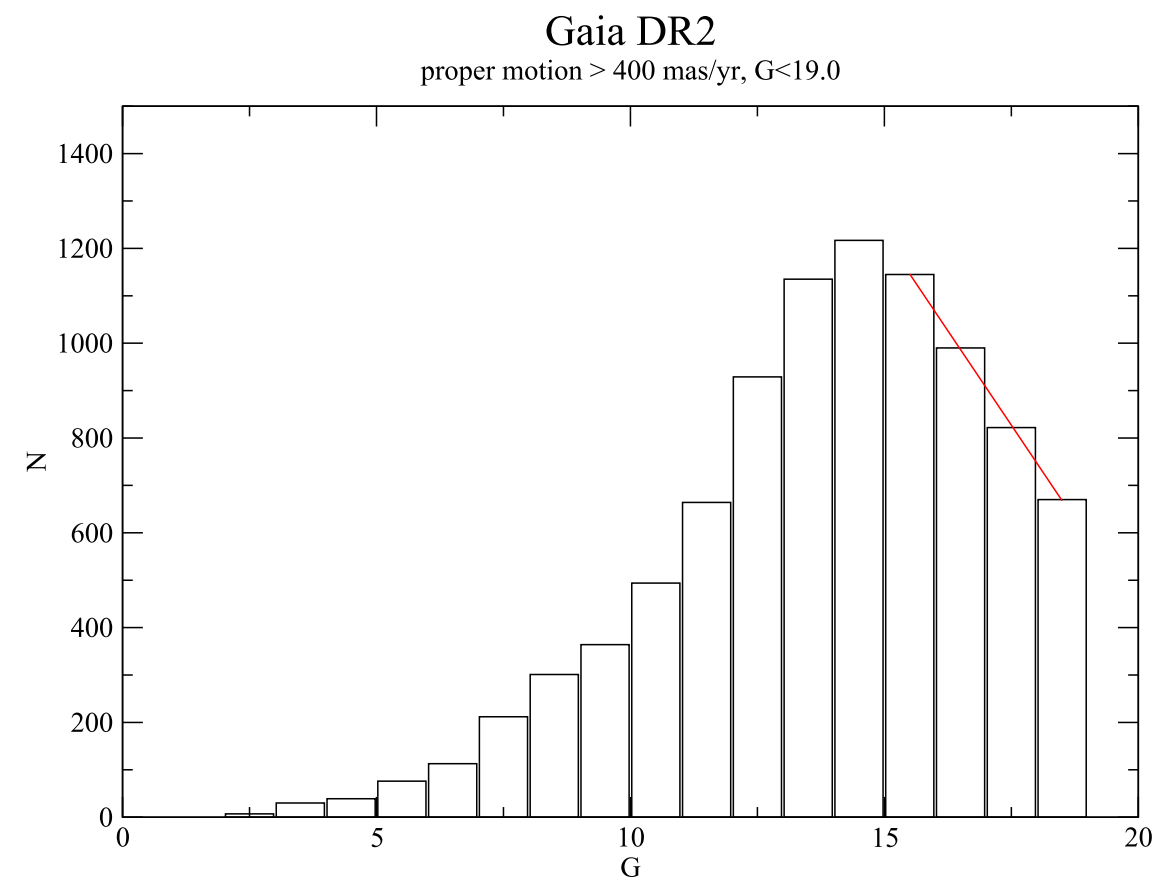

Figure 5. Proper motions in Gaia. We show all objects (9208) with high proper motions larger than 400 mas per year. Only 2482 are in the magnitude range $16<g<19$.

we list as "mismatches" together with the closest Pan-STARRS neighbor. Only the positional proximity is used. In this early study we covered only $60 \%$ of the sky (about 600 million USNO objects) due to limitations in computing time, and some regions are left out in the cross-match, as seen in Figure 4.

Using a $30^{\prime \prime}$ threshold (a limit set by the available computing time in the cloud environment), we find 426,975 mismatches (corresponding to a mismatch rate of $0.074 \%$ ). Correcting for differences in sky coverage between USNO and Pan-STARRS by removing all objects with decl. $<-30^{\circ}, 151,193$ of the mismatches can be considered for further investigation. The mismatch rate is within the range of various data processing artifacts existing in sky surveys, and among these artifacts we must search for real candidates.

\subsubsection{Treatment of High Proper Motion Objects}

For the 151,193 mismatches we first must ask: how many of these are only the result of a star just moving away over the last $70 \mathrm{yr}$ ? We approach the problem by estimating the number of objects that would escape our $30^{\prime \prime}$ cross-match radius. A $30^{\prime \prime}$ cross-match radius over a $70 \mathrm{yr}$ timeline translates to proper motions larger than 0"! 4 per year. We therefore use the Gaia Data Release 2 (DR2) catalog (Gaia Collaboration et al. 2018a, 2018b) to obtain all catalog objects that have $\mu_{\text {tot }}>0$ ". 4 per year. The catalog is complete down to $g \sim 19$. We plot a histogram of their magnitudes in Figure 5.

As we later find that $95 \%$ of the objects in our Mismatch Sample are fainter than mag 16 (see Figure 6), we estimate the number of objects with $g>16$ in Gaia DR2. There are 2482 

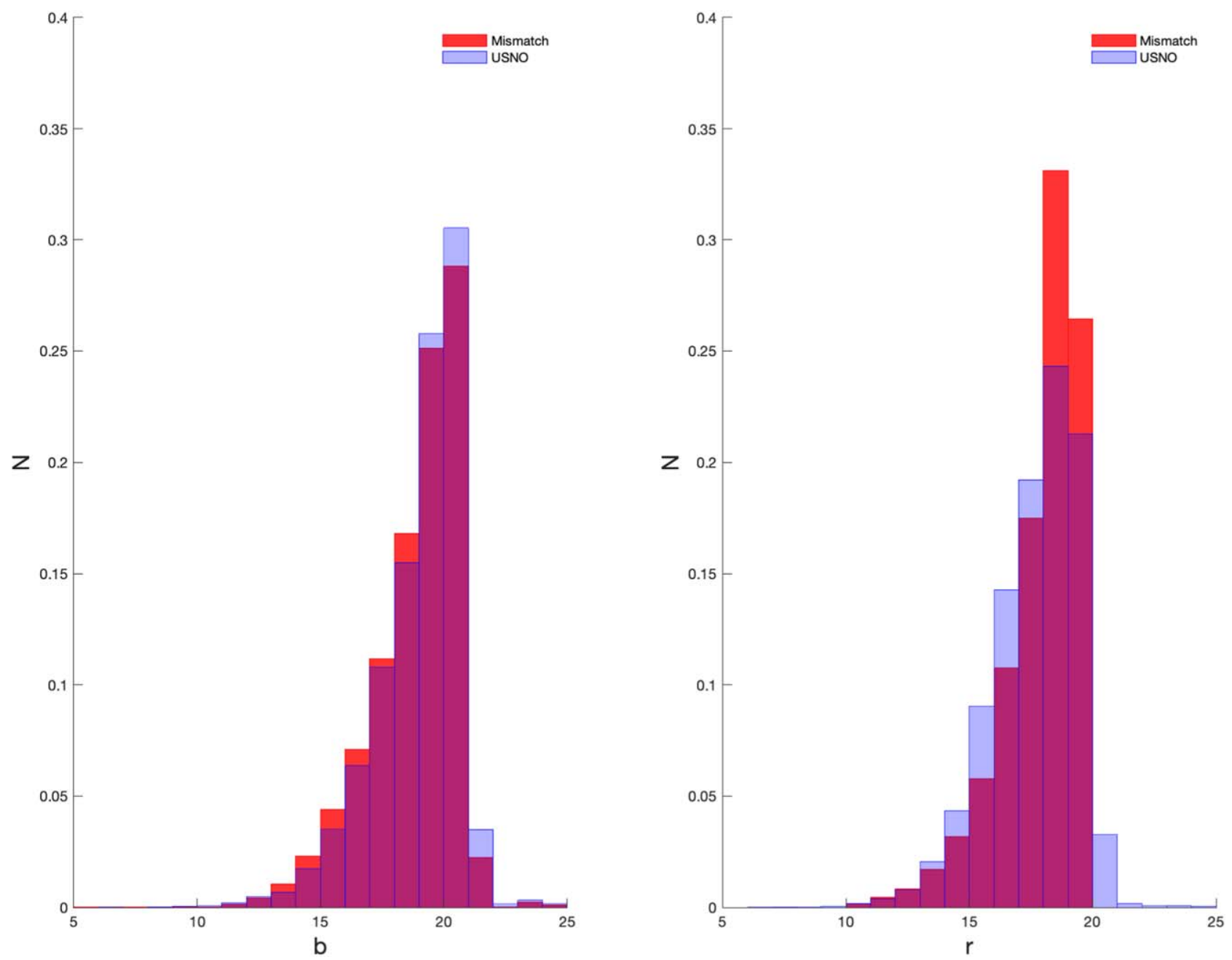

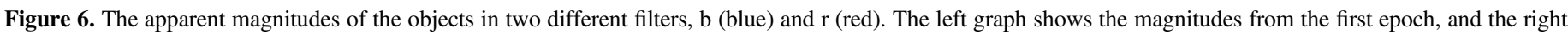
graph from the second epoch. The Mismatch Sample is compared to 50,000 randomly selected USNO objects. The histograms are normalized and zoomed.

objects in the range $16<g<19$. We extrapolate that the number of objects in the last bin $19<g<20$ is $\sim 500$, which means that the number of objects in Gaia DR2 with $g>16$ and proper motions larger than 0 " 4 per year is roughly $\sim 3000$. Correcting for the sky coverage used in our cross-match, this decreases the number by a factor of two, meaning that we may expect around $\sim 1500$ objects with high proper motion to contaminate our 150,000 mismatches. These objects can, however, be spotted when comparing the images and their surrounding fields.

We filter the mismatches by one additional limit on proper motion. This limit is set by the image field we are prepared to investigate visually later on. Any USNO star that moves away will move at a limited angle distance per unit time, and will be seen as an "appearing" case in the corresponding Pan-STARRS survey at a different location, likely with the same colors and magnitude (unless also variable).

The Gaia survey has shown that there are only nine stars known to us with proper motions larger than $5^{\prime \prime}$ per year. Over $70 \mathrm{yr}$ this corresponds to a movement of about $7^{\prime}$ between the POSS-I and Pan-STARRS images. For a radius of $7^{\prime}$ it would therefore be wise to use image fields of size $15^{\prime}$ when we compare the images, if we want to keep all objects with proper motions up to $5^{\prime \prime}$ per year. We note that USNO's proper motions carry much larger uncertainties than those of Gaia, and removing all objects with proper motions in USNO larger than $5^{\prime \prime}$ per year (about 130 objects in the Mismatch Sample) would leave us 151,063 objects.

For practical purposes, we shall use $5^{\prime} \times 5^{\prime}$ images (a search radius of 2.5 ). We filter the data so that we restrict the listed USNO proper motions to be less than 4 ". 3 per year, leaving 151,038 objects in our Mismatch Sample.

\subsection{Visually Inspecting a Subset with the SDSS}

One of the ways to investigate the 151,038 mismatches is to look at those missing in the SDSS Data Release 12 (DR12). The SDSS only covers the Northern Hemisphere, and therefore approximately half of the objects have not been observed in both surveys. Also, the SDSS started at an earlier epoch than Pan-STARRS, which reduces the time window for a potential disappearance by about $10 \mathrm{yr}$. Consequently, vanishing events that have happened in the last decade may remain undetected.

In order to cross-match with the SDSS DR12, we use the CasJobs interface, ${ }^{23}$ upload our coordinates to the server, and use the Footprint function to check whether a coordinate is within the SDSS scanned field. We see that 64,475 objects out of 151,038 can be found within the scanned field of the SDSS.

23 https://skyserver.sdss.org/CasJobs/ 

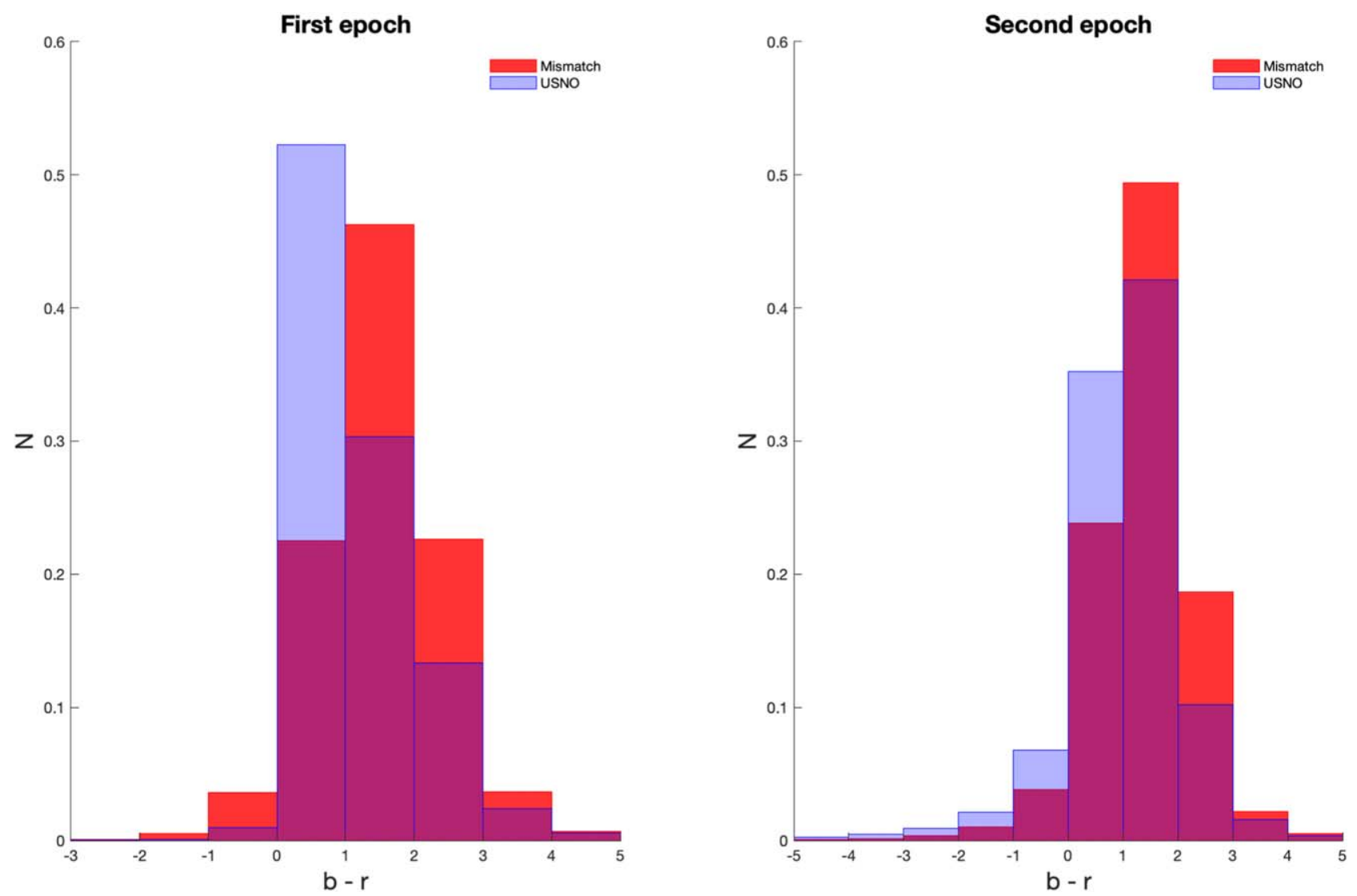

Figure 7. $b-r$ of the objects in two different filters, $b$ (blue) and $r$ (red). The left plot shows the colors from the first epoch, and the right plot those from the second epoch. The Mismatch Sample is compared to 50,000 randomly selected USNO objects. The histograms are normalized and zoomed.

These objects we re-upload to the CasJobs, and we then do a closest neighbor search with a radius of $0{ }^{\prime} 08\left(5^{\prime \prime}\right)$. About 23,667 objects have no detectable closest counterpart in this search zone. $^{24}$ This means that roughly one-third of our candidates remain when we use $5^{\prime \prime}$ as a cross-match radius.

Here, we carry out a similar analysis to that in Section 3.2.1, taking into account that SDSS only covers about one-fourth of the sky, and see that the estimated number of expected mismatches for proper motions necessary to exceed a $5^{\prime \prime}$ crossmatch $\left(\mu_{\mathrm{tot}}>00^{\prime \prime} 080\right.$ per year) is large-about 125,000 objects. However, most of these objects will not be a part of our visual subset. That previously used a $30^{\prime \prime}$ cross-match radius with Pan-STARRS.

At this point it would have been useful to employ image differencing software to compare the images to identify any obvious differences in pairs of very similar images. But, as we compare images made with widely different telescopes, instrumentation, and methods (photographic versus CCD), we do not gain much advantage by doing this step. Moreover, the hard drive space required to download the many fits files is prohibitive; 1000 images occupy $\sim 1 \mathrm{~TB}$. Therefore, we have inspected each of 23,667 candidates individually by visually comparing the images found in DSS $1,{ }^{25}$ STScI archive, ${ }^{26}$ and SDSS Explorer. ${ }^{27}$ First, we used the SDSS Explorer list-view to remove all objects that had an obvious flaw such as a bright

\footnotetext{
${ }^{24}$ Using the fGetNearestObjEq(m.ra,m.dec, 0.08$)$.

25 http://catserver.ing.iac.es/dss1/

26 https://archive.stsci.edu

${ }^{27}$ http://casjobs.sdss.org/dr15/en/tools/explore/Summary.aspx?
}

star or dead stripe in the SDSS image. See Villarroel et al. (2016) for details. This left 6359 objects, where no obvious flaw was causing the mismatch. In the next stage we individually examined the 6359 images in the DSS1 and only kept those that had an object in the center of the image, in order to remove false positives among the original USNO objects. This left 1691 candidates that had something clearly visible in the center of the DSS1 image. The SDSS subset effectively covers about 90 million stars from the USNO starting sample.

\section{Results from the New Searches}

\subsection{The Properties of the New "Mismatch Sample"}

One possibility is that the mismatches we have found represent objects with some typical problems. For instance, our objects could have larger average proper motion than reported in USNO. Also, our objects could have fewer detections associated with them, in comparison to the "average" USNO object, which leads to a number of false positives. Therefore, we investigate some basic properties of the Mismatch Sample, and compare them to 49,999 typical USNO objects, randomly selected from the entire USNO catalog.

Figure 6 shows histograms over the apparent magnitudes (blue and red bands) for the Mismatch Sample and the 50,000 randomly selected USNO objects. We see that the mean value in the blue band is $b \sim 18.85 \pm 0.01$ (Mismatch Sample) and $b \sim 19.01 \pm 0.01$ (USNO) in the first epoch (POSS-I: years 1949-1966). For the red band the average is $r \sim 17.86 \pm 0.004$ (Mismatch Sample) and $r \sim 17.72 \pm 0.01$ (USNO). A twosample Kolmogorov-Smirnov test reveals a small but 

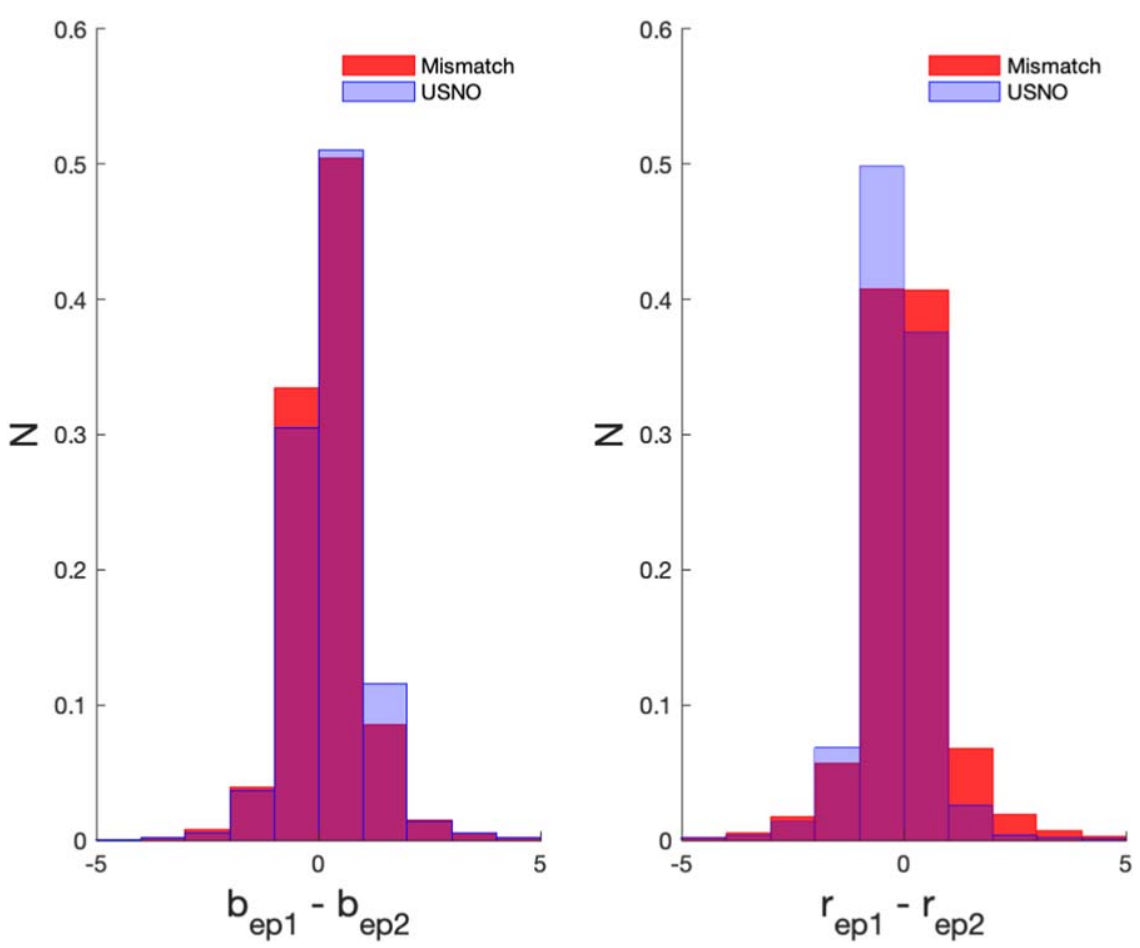

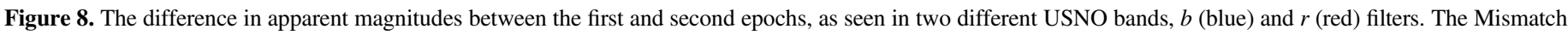
Sample is compared to 50,000 randomly selected USNO objects. The histograms are normalized and zoomed.

statistically significant difference between the samples, where the mismatch objects are slightly fainter in the red, but brighter in the blue band.

As a next step, we consider the colors. See Figure 7. The filters used, O (POSS-I blue), E (POSS-I red), J (POSS-II blue), and F (POSS-II red), have effective wavelengths of 4100, 6500, 4700, and $6600 \AA$. The samples could possibly come from two different color distributions. Indeed, a two-sample Kolmogorov-Smirnov test shows a statistically significant difference if testing with the nominal value of $\alpha<0.05$. The average colors in the first epoch are $b-r \sim 1.49 \pm 0.003$ (mismatch) and $b-r \sim 0.94 \pm 0.01$ (USNO sample). In the second epoch, the corresponding mean values are $b-r \sim 1.37 \pm 0.003$ (mismatch) and $b-r \sim$ $0.99 \pm 0.01$ (USNO sample). As the colors at the faint end may be uncertain, we also considered the corresponding color indices when using magnitudes brighter than 18 mag. We see that the average color differences in the first epoch are more pronounced for magnitudes $<18$, with $b-r \sim 1.22 \pm 0.005$ (mismatch) versus $b-r \sim 0.400 \pm 0.01$ (USNO sample).

We also compare variability separately in the two different bands. We show the difference between the brightness in the first and second epochs in Figure 8. In the blue band, the average change in magnitude is $0.19 \pm 0.004$ mag (mismatch) or $0.27 \pm 0.007$ (USNO). While the difference is significant enough to be noted in a Kolmogorov-Smirnov test, it is not particularly large and totally within the instrumental or calibration errors of USNO (Madsen \& Gaensler 2013). In the red band the difference is: $0.03 \pm 0.003$ mag (mismatch) and $-0.17 \pm 0.005 \mathrm{mag}$ (USNO). The difference here is also statistically significant in a two-sample Kolmogorov-Smirnov test. However, these differences are very likely to be the result of photometric calibration issues in the USNO survey, where the standard deviation of magnitudes in any band is about 0.3 mag but the systematic errors can be up to several magnitudes
(Monet et al. 2003; Madsen \& Gaensler 2013). These errors can happen, for instance, when measuring the magnitudes of objects in the neighborhood of very bright stars.

We have considered the mean proper motions (the absolute values) in our samples, from the square root of the sum of squares of $\mu_{\mathrm{R} . \mathrm{A}}$ and $\mu_{\mathrm{decl}}$. as listed in USNO. Figure 9 shows the proper motion distributions. We see that the mean $\mu_{\text {total }}$ is $76.7 \pm$ 0.55 mas $\mathrm{yr}^{-1}$ (Mismatch Sample) and $33.0 \pm 0.51$ mas $\mathrm{yr}^{-1}$ (USNO sample). The $\mu_{\text {total }}$ differs significantly and by a factor of $\sim 2$, where the mismatch objects have higher proper motions than the typical USNO objects.

Interestingly, the objects in the Mismatch Sample show a larger number of detections, $\sim 3.8$ per object compared to the average of $\sim 3.5$ detections per object in the USNO sample. See Figure 10.

Summing up, we have learned that the objects we find as mismatches are in general redder and have higher proper motions. This means that nearby $(<100 \mathrm{pc})$ red stars could be significant contributors to the $\sim 150,000$ Mismatch Sample. For instance, $\mathrm{M}$ dwarfs with magnetic flares could be among these. But what we see may also mean that the different detections for "one" USNO object may correspond to different objects, which happen to be close to each other by chance.

\subsection{The Visually Inspected Sample}

We examine the final 1691 candidates and compare the old and new images between the DSS1 and the SDSS, complementing the study with images in several bands in the STScI archive when the DSS1 images were not clear enough. At this stage, most of the candidates are the result of slightly offset coordinates, and the images reveal that the objects are present in both old and new images, with tiny offsets of the central point. About 200 of the 1691 candidates are caused by dead stripes in the SDSS. Finally, about 100 candidates remain, most 


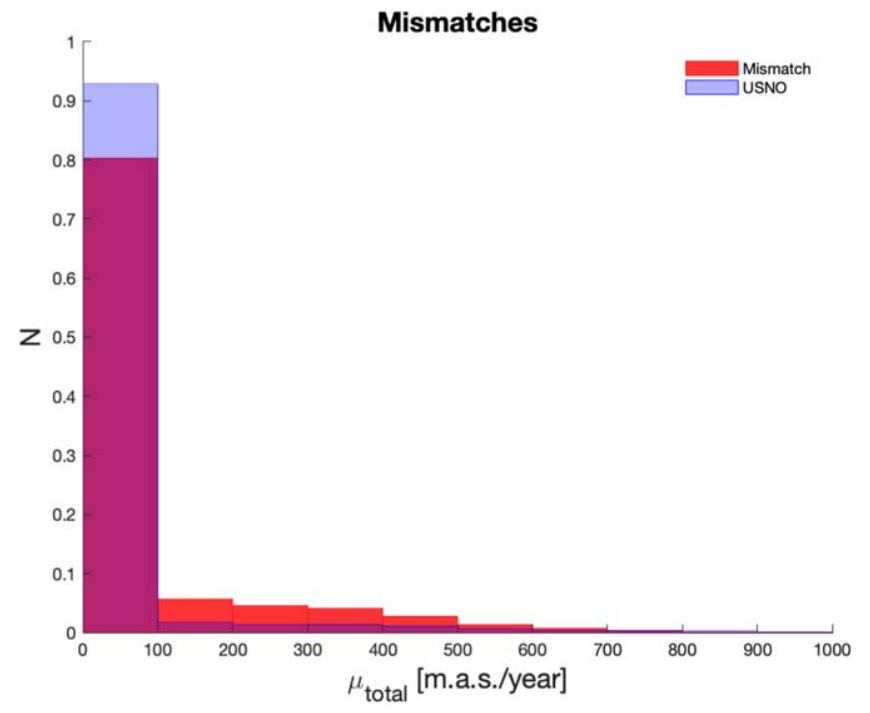

Figure 9. The USNO proper motions $\mu_{\text {total }}$. The Mismatch Sample is compared to 50,000 randomly selected USNO objects. The histograms are normalized.

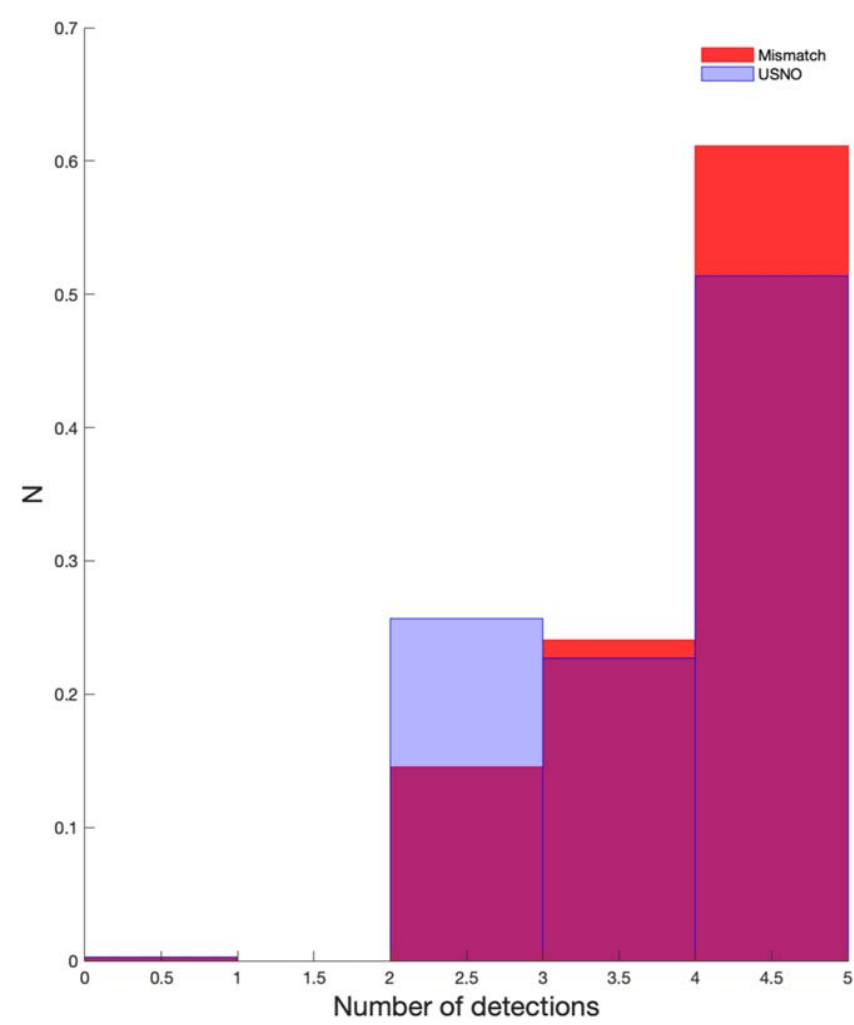

Figure 10. The total number of detections. The Mismatch Sample is compared to 50,000 randomly selected USNO objects. Objects in the Mismatch Sample have, on average, a higher number of detections than typical USNO objects. The histograms are normalized and zoomed.

with a point-like appearance. Nearly all these candidates are single-epoch observations in the POSS-I, red band. This is likely due to the way the USNO catalog was constructed, but possibly also due to the order of visual inspection, where we started by first examining the POSS-I red images, later the POSS-I blue, etc., which could introduce a bias in favor of detecting these one-time events in the POSS-I red band.

One possible way of weeding out plate flaws using only the digital scans is by examining the PSFs of stars with a similar magnitude range on each plate, and comparing them to the PSF measured for each candidate. Using DS9 we measured the radial profile of the light distribution near a typical star, where the width of the PSF is estimated to be the FWHM of the Gaussian. The sharpness of the stars varies somewhat between the plates, but when we compare the FWHM of the given transient on a plate with the FWHM of a well-known star on the same plate, we find that the full widths at half maximum are similar in most cases. However, about 20 objects need to be removed because either they appear asymmetric or their widths are significantly smaller than those of real stars. We find that some typical artifacts have an FWHM significantly larger than normal stars, and we therefore remove candidates with significantly larger PSFs as well. However, we keep candidates that look like binary stars or multiple star systems, even if these could well be artifacts.

We list the $\sim 100$ surviving objects (preliminary candidates) in Table 2. No candidate has a cross-match within $30^{\prime \prime}$ of a source in the General Catalog of Variable Stars (Samus et al. 2017), which means none of them is an already known variable object.

In a separate article (B. Villarroel et al. 2019, in preparation) we examine each surviving object in depth with the aim of identifying the true nature of our sources and selecting the top candidates. As individual examples, we include images of typical objects that only are seen in one epoch. See Figures 11 and 12. The latter candidate stands out among the others. We see how something is visible in the POSS-I and POSS-II red filters, but with a slight shift. In the more recent images from SDSS and Pan-STARRS nothing is visible, as can be seen in the figure. However, one must take into account the exact location, the signal-to-noise ratio of the detections, and the elongated fiber-like structure next to the two stars in POSS-I (possibly an artifact?). This needs further investigation. While the blue POSS filters are not shown here, there is possibly also an extremely faint detection in the POSS-I blue filter, but nothing at all visible in the POSS-II blue.

\section{Discussion}

The VASCO project aims to look for vanishing and appearing objects using old and new sky surveys. In 2016 we performed a pilot study (Villarroel et al. 2016) and searched for vanishing stars in a cross-match of 10 million objects with no proper motion in USNO and SDSS (since 2000). We found one point source and established that the probability of discovering vanishing events was about one in 10 million (or less) within this timeframe of roughly a decade.

We have now performed a follow-up analysis of the old images, more archival searches, and new observations of this object from Teide Observatory and the Nordic Optical Telescope. Near the original location, within 2!"4 and 1".4, respectively, two objects that are approximately 4-4.5 mag fainter can be found in the red band.

We conclude that there are four possibilities:

1. The detection is a variable object that has dropped approximately 4-4.5 mag between the 1950s and 2018 .

2. The detection is a very red (or redshifted) transient event that happened in 1950 March. It could have been an M dwarf that flared during the POSS-I exposure.

3. The object is a plate scratch. This appears unlikely due to the point-like nature of the detection itself. 
Table 2

Coordinates (J2000.0) of the First Set of $\sim 100$ Surviving Candidates

\begin{tabular}{|c|c|c|c|c|c|c|c|c|}
\hline R.A. & Decl. & $r$ (mag) & R.A. & Decl. & $r$ (mag) & R.A. & Decl. & $r$ (mag) \\
\hline $00^{\mathrm{h}} 11^{\mathrm{m}} 19^{\mathrm{s}} .43$ & $-03^{\circ} 09^{\prime} 45^{\prime \prime} 22$ & 19.18 & $10^{\mathrm{h}} 03^{\mathrm{m}} 33^{\mathrm{s}} .36$ & $+22^{\circ} 09^{\prime} 01^{\prime \prime} 65$ & 19.27 & $12^{\mathrm{h}} 38^{\mathrm{m}} 21^{\mathrm{s}} .48$ & $+42^{\circ} 45^{\prime} 09^{\prime \prime} 17$ & 19.26 \\
\hline $00^{\mathrm{h}} 13^{\mathrm{m}} 53^{\mathrm{s}} .05$ & $+03^{\circ} 23^{\prime} 20^{\prime \prime} .51$ & 19.32 & $10^{\mathrm{h}} 03^{\mathrm{m}} 39^{\mathrm{s}} .53$ & $+16^{\circ} 45^{\prime} 01{ }^{\prime \prime} 19$ & 17.94 & $12^{\mathrm{h}} 42^{\mathrm{m}} 11^{\mathrm{s}} .06$ & $+16^{\circ} 08^{\prime} 55^{\prime \prime} 03$ & 13.57 \\
\hline $00^{\mathrm{h}} 15^{\mathrm{m}} 34^{\mathrm{s}} .89$ & $+17^{\circ} 35^{\prime} 24^{\prime \prime} .87$ & 19.09 & $10^{\mathrm{h}} 09^{\mathrm{m}} 55^{\mathrm{s}} .66$ & $+14^{\circ} 45^{\prime} 07^{\prime \prime} .56$ & 14.45 & $12^{\mathrm{h}} 53^{\mathrm{m}} 46^{\mathrm{s}} .42$ & $+27^{\circ} 21^{\prime} 08^{\prime \prime} \cdot 75$ & 18.13 \\
\hline $00^{\mathrm{h}} 37^{\mathrm{m}} 25^{\mathrm{s}} \cdot 41$ & $+27^{\circ} 12^{\prime} 34^{\prime \prime} .53$ & 19.36 & $10^{\mathrm{h}} 18^{\mathrm{m}} 59^{\mathrm{s}} \cdot 33$ & $+21^{\circ} 14^{\prime} 13^{\prime \prime} 16$ & 19.38 & $13^{\mathrm{h}} 08^{\mathrm{m}} 16^{\mathrm{s}} \cdot 49$ & $+18^{\circ} 46^{\prime} 48^{\prime \prime} 79$ & 19.10 \\
\hline $00^{\mathrm{h}} 41^{\mathrm{m}} 22.82$ & $+03^{\circ} 22^{\prime} 04^{\prime \prime} 51$ & 19.42 & $10^{\mathrm{h}} 19^{\mathrm{m}} 27^{\mathrm{s}} \cdot 62$ & $+15^{\circ} 03^{\prime} 17^{\prime \prime} 82$ & 18.89 & $13^{\mathrm{h}} 30^{\mathrm{m}} 37^{\mathrm{s}} \cdot 34$ & $+15^{\circ} 04^{\prime} 19^{\prime \prime} 70$ & 19.32 \\
\hline $00^{\mathrm{h}} 42^{\mathrm{m}} 38^{\mathrm{s}} \cdot 75$ & $+12^{\circ} 30^{\prime} 47^{\prime \prime} .66$ & 18.40 & $10^{\mathrm{h}} 22^{\mathrm{m}} 04^{\mathrm{s}} .56$ & $+24^{\circ} 26^{\prime} 28^{\prime \prime} .03$ & 18.90 & $13^{\mathrm{h}} 30^{\mathrm{m}} 54^{\mathrm{s}} .17$ & $+02^{\circ} 30^{\prime} 07^{\prime \prime} 78$ & 19.05 \\
\hline $01^{\mathrm{h}} 39^{\mathrm{m}} 29^{\mathrm{s}} .57$ & $+09^{\circ} 00^{\prime} 39^{\prime \prime} .28$ & 19.38 & $10^{\mathrm{h}} 40^{\mathrm{m}} 48^{\mathrm{s}} .43$ & $+21^{\circ} 53^{\prime} 28^{\prime \prime} 36$ & 19.36 & $13^{\mathrm{h}} 55^{\mathrm{m}} 01^{\mathrm{s}} .30$ & $+08^{\circ} 10^{\prime} 42^{\prime \prime} 56$ & 19.36 \\
\hline $01^{\mathrm{h}} 55^{\mathrm{m}} 30^{\mathrm{s}} \cdot 70$ & $+09^{\circ} 00^{\prime} 42^{\prime \prime} 19$ & 18.82 & $10^{\mathrm{h}} 43^{\mathrm{m}} 14^{\mathrm{s}} \cdot 40$ & $+17^{\circ} 10^{\prime} 52^{\prime \prime} 50$ & 18.99 & $13^{\mathrm{h}} 55^{\mathrm{m}} 06^{\mathrm{s}} \cdot 31$ & $+11^{\circ} 11^{\prime} 46^{\prime \prime} 36$ & 17.51 \\
\hline $01^{\mathrm{h}} 56^{\mathrm{m}} 36^{\mathrm{s}} \cdot 11$ & $+22^{\circ} 52^{\prime} 08^{\prime \prime} 83$ & 16.91 & $10^{\mathrm{h}} 51^{\mathrm{m}} 35^{\mathrm{s}} .73$ & $+15^{\circ} 13^{\prime} 38^{\prime \prime} 71$ & 18.91 & $14^{\mathrm{h}} 10^{\mathrm{m}} 38^{\mathrm{s}} .04$ & $+26^{\circ} 00^{\prime} 33$ " 45 & 19.02 \\
\hline $02^{\mathrm{h}} 12^{\mathrm{m}} 54^{\mathrm{s}} .67$ & $+06^{\circ} 51^{\prime} 45^{\prime \prime} .29$ & 16.24 & $10^{\mathrm{h}} 55^{\mathrm{m}} 38^{\mathrm{s}} .33$ & $+14^{\circ} 55^{\prime} 38^{\prime \prime} 75$ & 18.54 & $14^{\mathrm{h}} 11^{\mathrm{m}} 43^{\mathrm{s}} \cdot 63$ & $+26^{\circ} 49^{\prime} 39^{\prime \prime} 07$ & 18.10 \\
\hline $02^{\mathrm{h}} 53^{\mathrm{m}} 01^{\mathrm{s}} .89$ & $-01^{\circ} 40^{\prime} 11^{\prime \prime} 68$ & 18.54 & $11^{\mathrm{h}} 07^{\mathrm{m}} 51^{\mathrm{s}} .02$ & $+35^{\circ} 02^{\prime} 14^{\prime \prime} 50$ & 19.23 & $14^{\mathrm{h}} 11^{\mathrm{m}} 57^{\mathrm{s}} .26$ & $+12^{\circ} 33^{\prime} 49^{\prime \prime} 79$ & 18.66 \\
\hline $03^{\mathrm{h}} 31^{\mathrm{m}} 34^{\mathrm{s}} .89$ & $+05^{\circ} 24^{\prime} 46^{\prime \prime} 04$ & 18.47 & $11^{\mathrm{h}} 07^{\mathrm{m}} 53^{\mathrm{s}} .57$ & $+18^{\circ} 50^{\prime} 28^{\prime \prime}$. 18 & 18.38 & $14^{\mathrm{h}} 22^{\mathrm{m}} 50^{\mathrm{s}} \cdot 45$ & $+44^{\circ} 21^{\prime} 32^{\prime \prime} 54$ & 18.90 \\
\hline $08^{\mathrm{h}} 21^{\mathrm{m}} 44^{\mathrm{s}} \cdot 71$ & $+58^{\circ} 18^{\prime} 12^{\prime \prime} 27$ & 19.01 & $11^{\mathrm{h}} 32^{\mathrm{m}} 16^{\mathrm{s}} \cdot 08$ & $+02^{\circ} 34^{\prime} 24^{\prime \prime} 31$ & 19.03 & $15^{\mathrm{h}} 22^{\mathrm{m}} 30^{\mathrm{s}} \cdot 96$ & $+24^{\circ} 40^{\prime} 05^{\prime \prime} 99$ & 17.62 \\
\hline $08^{\mathrm{h}} 22^{\mathrm{m}} 16^{\mathrm{s}} \cdot 49$ & $+37^{\circ} 46^{\prime} 05^{\prime \prime} .12$ & 18.27 & $11^{\mathrm{h}} 32^{\mathrm{m}} 25^{\mathrm{s}} \cdot 37$ & $+05^{\circ} 22^{\prime} 57^{\prime \prime} 00$ & 19.21 & $15^{\mathrm{h}} 29^{\mathrm{m}} 41^{\mathrm{s}} .43$ & $+22^{\circ} 58^{\prime} 18^{\prime \prime} 19$ & 16.18 \\
\hline $08^{\mathrm{h}} 29^{\mathrm{m}} 44^{\mathrm{s}} \cdot 74$ & $+61^{\circ} 29^{\prime} 34^{\prime \prime} .84$ & 18.95 & $11^{\mathrm{h}} 49^{\mathrm{m}} 31^{\mathrm{s}} \cdot 70$ & $+16^{\circ} 07^{\prime} 17^{\prime \prime} 33$ & 17.05 & $15^{\mathrm{h}} 38^{\mathrm{m}} 31^{\mathrm{s}} \cdot 44$ & $+43^{\circ} 02^{\prime} 04^{\prime \prime} 96$ & 17.57 \\
\hline $08^{\mathrm{h}} 35^{\mathrm{m}} 18^{\mathrm{s}} \cdot 72$ & $+62^{\circ} 03^{\prime} 51^{\prime \prime} 51$ & 19.43 & $11^{\mathrm{h}} 53^{\mathrm{m}} 31^{\mathrm{s}} \cdot 90$ & $+63^{\circ} 03^{\prime} 59^{\prime \prime} 36$ & 19.43 & $15^{\mathrm{h}} 41^{\mathrm{m}} 04^{\mathrm{s}} .78$ & $+09^{\circ} 00^{\prime} 54^{\prime \prime} 50$ & 16.49 \\
\hline $08^{\mathrm{h}} 44^{\mathrm{m}} 04^{\mathrm{s}} .68$ & $+57^{\circ} 56^{\prime} 55^{\prime \prime} 54$ & 17.84 & $11^{\mathrm{h}} 56^{\mathrm{m}} 22^{\mathrm{s}} .08$ & $+67^{\circ} 04^{\prime} 36^{\prime \prime} 33$ & 19.49 & $15^{\mathrm{h}} 48^{\mathrm{m}} 55^{\mathrm{s}} .08$ & $+62^{\circ} 24^{\prime} 52^{\prime \prime} 38$ & 19.25 \\
\hline $08^{\mathrm{h}} 51^{\mathrm{m}} 17^{\mathrm{s}} .30$ & $+62^{\circ} 58^{\prime} 36^{\prime \prime} 16$ & 18.91 & $12^{\mathrm{h}} 17^{\mathrm{m}} 49^{\mathrm{s}} .03$ & $+67^{\circ} 40^{\prime} 27^{\prime \prime} 40$ & 19.27 & $16^{\mathrm{h}} 09^{\mathrm{m}} 45^{\mathrm{s}} \cdot 19$ & $+38^{\circ} 34^{\prime} 48^{\prime \prime} 68$ & 18.85 \\
\hline $09^{\mathrm{h}} 18^{\mathrm{m}} 46^{\mathrm{s}} .22$ & $+62^{\circ} 29^{\prime} 49^{\prime \prime} .10$ & 18.60 & $12^{\mathrm{h}} 23^{\mathrm{m}} 15^{\mathrm{s}} .99$ & $+16^{\circ} 28^{\prime} 14^{\prime \prime} .92$ & 19.23 & $16^{\mathrm{h}} 10^{\mathrm{m}} 01^{\mathrm{s}} .20$ & $+61^{\circ} 31^{\prime} 11^{\prime \prime} 25$ & 17.79 \\
\hline $09^{\mathrm{h}} 33^{\mathrm{m}} 09^{\mathrm{s}} .84$ & $+15^{\circ} 08^{\prime} 35^{\prime \prime} 99$ & 19.17 & $12^{\mathrm{h}} 29^{\mathrm{m}} 37^{\mathrm{s}} .58$ & $+61^{\circ} 17^{\prime} 59^{\prime \prime} 97$ & 18.44 & $16^{\mathrm{h}} 24^{\mathrm{m}} 00^{\mathrm{s}} \cdot 31$ & $+61^{\circ} 21^{\prime} 00^{\prime \prime} 26$ & 18.21 \\
\hline $09^{\mathrm{h}} 36^{\mathrm{m}} 21^{\mathrm{s}} \cdot 19$ & $+37^{\circ} 49^{\prime} 43^{\prime \prime} 86$ & 18.82 & $12^{\mathrm{h}} 30^{\mathrm{m}} 28^{\mathrm{s}} .80$ & $+20^{\circ} 43^{\prime} 00^{\prime \prime} 95$ & 16.95 & $16^{\mathrm{h}} 34^{\mathrm{m}} 25^{\mathrm{s}} .99$ & $+34^{\circ} 04^{\prime} 24^{\prime \prime} 45$ & 17.40 \\
\hline $09^{\mathrm{h}} 39^{\mathrm{m}} 28^{\mathrm{s}} .66$ & $+22^{\circ} 39^{\prime} 41^{\prime \prime} 73$ & 19.48 & $12^{\mathrm{h}} 31^{\mathrm{m}} 55^{\mathrm{s}} .13$ & $-02^{\circ} 06^{\prime} 22^{\prime \prime} 03$ & 18.24 & $17^{\mathrm{h}} 20^{\mathrm{m}} 34^{\mathrm{s}} .85$ & $+44^{\circ} 00^{\prime} 11^{\prime \prime} 34$ & 19.49 \\
\hline $09^{\mathrm{h}} 42^{\mathrm{m}} 00^{\mathrm{s}} .89$ & $+16^{\circ} 55^{\prime} 56^{\prime \prime} 32$ & 18.86 & $12^{\mathrm{h}} 33^{\mathrm{m}} 09^{\mathrm{s}} .27$ & $+43^{\circ} 14^{\prime} 07^{\prime \prime} 05$ & 19.37 & $17^{\mathrm{h}} 25^{\mathrm{m}} 44^{\mathrm{s}} \cdot 62$ & $+73^{\circ} 54^{\prime} 09^{\prime \prime} 76$ & 19.39 \\
\hline $09^{\mathrm{h}} 43^{\mathrm{m}} 07^{\mathrm{s}} .39$ & $+31^{\circ} 11^{\prime} 59^{\prime \prime} 38$ & 18.77 & $12^{\mathrm{h}} 37^{\mathrm{m}} 23^{\mathrm{s}} .88$ & $+05^{\circ} 26^{\prime} 29^{\prime \prime} 40$ & 19.33 & $18^{\mathrm{h}} 30^{\mathrm{m}} 58^{\mathrm{s}} \cdot 12$ & $+40^{\circ} 54^{\prime} 00^{\prime \prime} 14$ & 18.55 \\
\hline
\end{tabular}

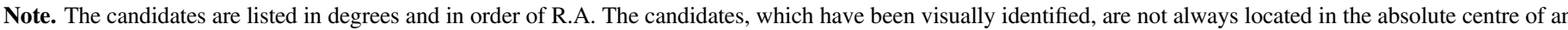

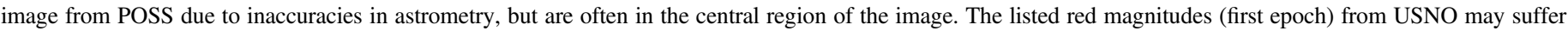
from large uncertainties due to the issues in photometry.

4. The object is a nearby, red, faint low-mass star or brown dwarf with a very high proper motion that has allowed the it to move 4.5 over a time span of $70 \mathrm{yr}$. As there are few stars with such high proper motion in USNO, this appears not too likely either.

Since only one secure detection of these objects exists, and although that detection seems to be of a point source, it is difficult to establish its nature.

We have performed a new, deeper cross-match of 600 million objects from USNO and the entire Pan-STARRS DR1 (starting in 2013) to search for more convincing vanished candidates, which supersedes the previous USNO sample by a factor of 60 . As Pan-STARRS goes deeper than SDSS, the new cross-match therefore allows us to exclude a large number of variable objects near the detection limit. We obtain a final sample of about 150,000 mismatches (the "Mismatch Sample") characterized by the lack of a counterpart in Pan-STARRS. We have investigated the properties of the Mismatch Sample and found that the mismatches are generally redder, more variable in the red band, and have higher average proper motion. Many of these could be $\mathrm{M}$ dwarfs closer than $100 \mathrm{pc}$, and if an $\mathrm{M}$ dwarf was flaring during the POSS-I exposure, it could be invisible in the PanSTARRS and SDSS surveys.

Cross-matching the Mismatch Sample with the SDSS, we find that 23,667 objects cannot be found in SDSS. The number of USNO objects surveyed by this cross-match is about 91 million. We examined each of these candidates in this subset visually. Most are artifacts of various sorts. However, about 100 candidates are point sources visible only in the photographic POSS-I plates that were taken from the 1950s to 1970 s. That means that the complete Mismatch Sample should contain at least 700 detections of this class. This is a significantly larger number than the eight known objects in our Galaxy that have proper motion larger than $5^{\prime \prime} \mathrm{yr}^{-1}$. A mismatch sample utilizing a $5^{\prime \prime}$ cross-match radius instead of the $30^{\prime \prime}$ cross-match radius currently used is expected to provide even more potential detections.

With the visual inspection performed on a subset of the Mismatch Sample (see Section 3.3) we have considered the most interesting candidates from a sample of roughly 90 million USNO objects. Among these, no truly vanishing star was convincingly detected, which means that we can expect the chance of finding vanishing-star events during $70 \mathrm{yr}$ to be less than 1 in 90 million in our Galaxy. In the Appendix we demonstrate with theoretical 


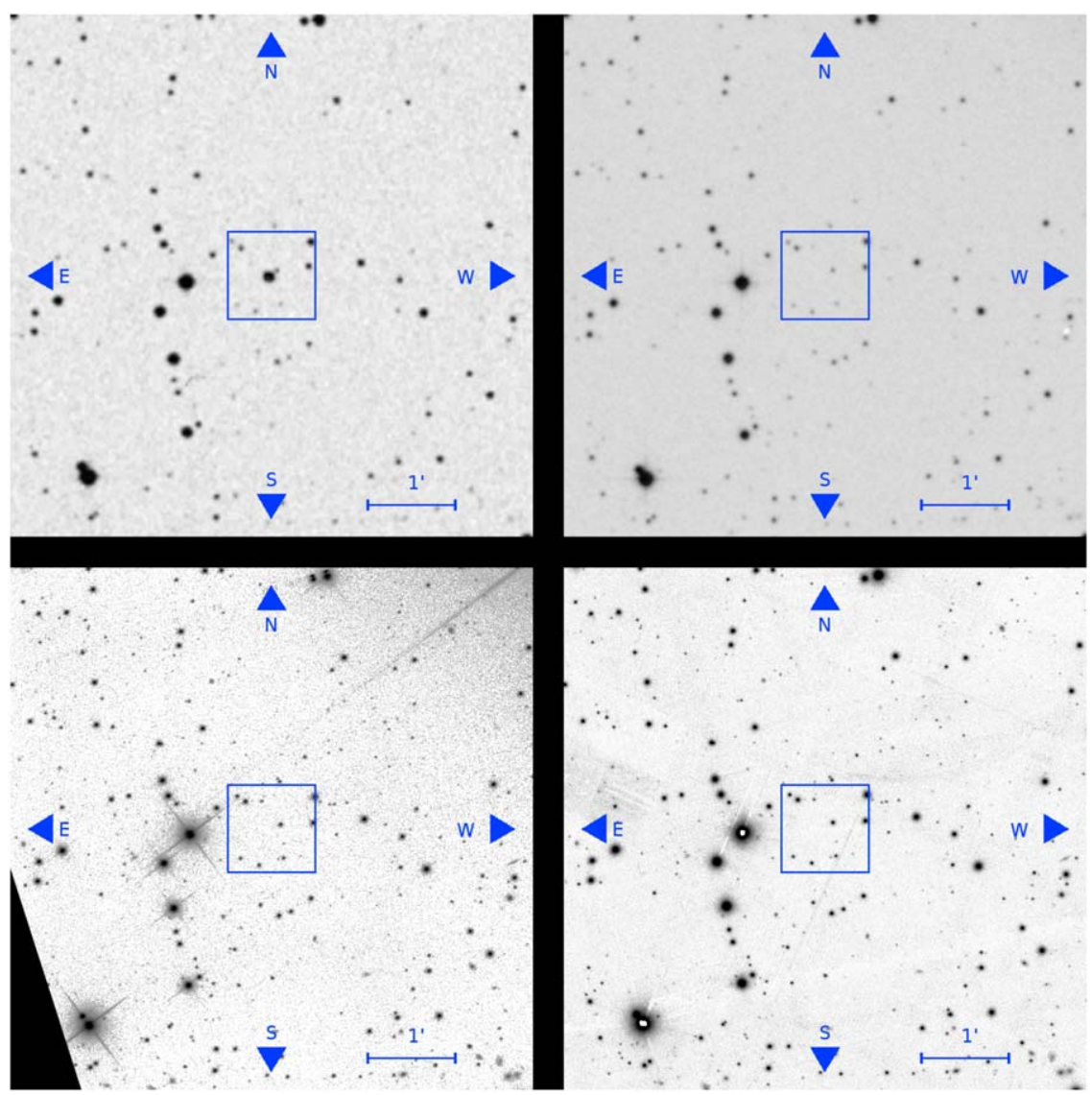

Figure 11. Example of one candidate shown in Table 2. We show the images from (upper left) POSS-I E red, (upper right) POSS-II red, (lower left) combination of SDSS filters, (lower right) Pan-STARRS $r$. The object is seen in the POSS-I red band and has the coordinates (R.A., decl.) $=277.7422$, 40.90004. Afterwards, it seems to have "vanished." Fine-centering the coordinates of this object gives (R.A., decl.) $=277.734042,40.9054433$.

calculations that one is not likely to encounter a failed supernova in the VASCO searches.

\subsection{One Hundred Red Events?}

What do we actually know about the transients? For the object in Villarroel et al. (2016), we can use the USNO limiting magnitude $b \sim 21$ to set a lower limit of the color, $b-r \geqslant 1.3$. But many other events in Table 2 appear to have much redder colors with $b-r \geqslant 7.4$, which may mean that our objects are a mixture of apparently red events. For some objects the red and blue observations might have happened simultaneously, while for others there may have been a significant offset in time between the red and blue observations. We note the similarity of the object in Villarroel et al. (2016) and the nuclear transient reported in Figure 6 of Djorgovski et al. (2001), where an event with $r \sim 18.5$ is observed in its bright phase in one red image, and only seems to be "extremely faint" in two other filters while revealing a background galaxy at $z \sim 1$ with $r \sim 24.5$. In our case, the NOT image reveals two background objects within angular distances of 1.4 and $2 . " 4,{ }^{28}$ close to the original spot. They too could be galaxies at high redshift. However, the offset in position between the USNO and NOT detections, which is caused either by the low resolution of POSS-I or by proper motion, puts this explanation in doubt.

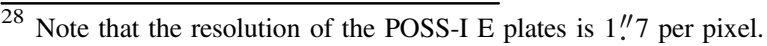

Most of the 100 events could be detected in one image and not be detected again. If one assumes that most of these events were detected in two filters at the same time, they are unusually red to be solar system objects, with half of the objects having colors $b-r \geqslant 2$. Solar system objects are typically much bluer (due to the color of reflected sunlight), even if rare exceptions exist. Taking Figure 11 as a typical example, the POSS-I red-band and blue-band images were obtained about a quarter of an hour apart according to the listed epochs for each image in the DSS plate finder. ${ }^{29}$ The exposure time for the red image is about 50 minutes. If the object were an asteroid and was quickly moving through the field of the red image in a few minutes, then it would be elongated on the plate. However, this object is point-like. In addition, the candidate is anomalously red and not seen in the blue band, which further decreases the likelihood that it is an asteroid. We have far too many candidates for nearby stars with high proper motion to comprise most of our sample. From the Gaia survey we know that there exist only eight stars with proper motions larger than $5^{\prime \prime}$ per year, which is the minimum proper motion needed to explain the "vanishing" events. Therefore, it is unlikely that many of the 100 events are objects with high proper motion.

Other events we may have observed are novae, supernovae at high redshift, and microlensing events or flares from $\mathrm{M}$ dwarfs. Some of the red transients might be intermediateluminosity red transients (Bond et al. 2009) or tidal disruption

${ }^{29}$ https://archive.stsci.edu/cgi-bin/dss_plate_finder 


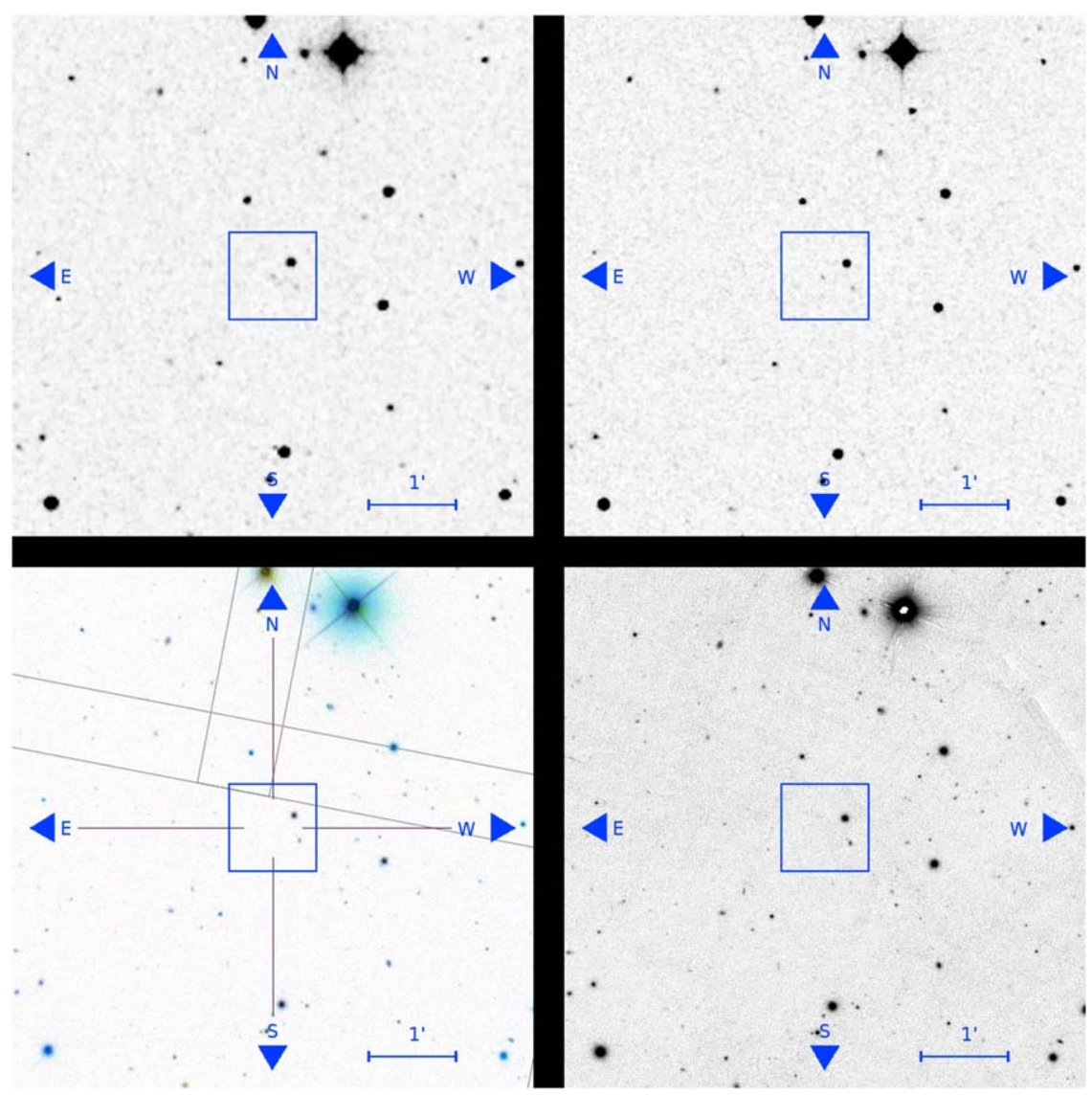

Figure 12. Note the bright star in the center in all images. We show the images from (upper left) POSS-I E red, (upper right) POSS-II red, (lower left) SDSS, (lower right) Pan-STARRS $r$. In the upper two images (POSS-I and POSS-II red filters), both the faint stars are present. In the lower two images (SDSS and Pan-STARRS r), only the right faint star is present.

events. Some of our candidates might be M-dwarf flares, because many of our objects are faint $(r \sim 18-19)$, red, and appear to have non-zero proper motion. $\mathrm{M}$ dwarfs tend to brighten several magnitudes during a flare, and recently a flare of $10 \mathrm{mag}$ was reported (Rodriguez et al. 2018).

We examined the digitized photographic plates for typical plate flaws in Section 4.2, and the objects listed in Table 2 have satisfied the selection criteria. We propose that these objects may be worth following up with transient sky surveys to see whether they might be recovered. We will analyze each of these 100 sources in a separate paper and attempt to carry out deep imaging of them.

Following the VASCO criteria introduced at the end of Section 1, we define the most interesting candidates as either single-time transients with large amplitudes $\Delta m>5$ mag or objects that were detected in more than one image prior to "disappearance." These objects are listed in Table 3. The candidates displayed in Figures 11 and 12 belong to this table.

\subsection{Implications for SETI Research}

The Search for Extraterrestrial Intelligence (SETI) nowadays includes a broad set of activities, where the two large domains of searches are done both in the radio and in the optical, in hopes of finding so-called "technosignatures" like those we ourselves are already capable of producing, such as interstellar communication with lasers (Schwartz \& Townes 1961). Optical SETI searches looking for lasers are particularly interesting, because these signatures often have a low temporal dispersion
Table 3

The Most Interesting Candidates

\begin{tabular}{|c|c|c|c|}
\hline R.A. & Decl. & R.A. & Decl. \\
\hline $00^{\mathrm{h}} 16^{\mathrm{m}} 06^{\mathrm{s}} .92$ & $+21^{\circ} 58^{\prime} 47^{\prime \prime} 82$ & $11^{\mathrm{h}} 56^{\mathrm{m}} 18^{\mathrm{s}} .10$ & $+67^{\circ} 04^{\prime} 23$ !' 15 \\
\hline $00^{\mathrm{h}} 42^{\mathrm{m}} 38^{\mathrm{s}} .77$ & $+12^{\circ} 30^{\prime} 36^{\prime \prime} 75$ & $12^{\mathrm{h}} 31^{\mathrm{m}} 55^{\mathrm{s}} .05$ & $-02^{\circ} 06^{\prime} 28^{\prime \prime} 11$ \\
\hline $00^{\mathrm{h}} 58^{\mathrm{m}_{3}} 32^{\mathrm{s}} \cdot 63$ & $+17^{\circ} 48^{\prime} 19^{\prime \prime} 44$ & $12^{\mathrm{h}} 42^{\mathrm{m}} 11^{\mathrm{s}} .02$ & $+16^{\circ} 08^{\prime} 57^{\prime \prime} \cdot 25$ \\
\hline $01^{\mathrm{h}} 56^{\mathrm{m}} 36^{\mathrm{s}} .27$ & $+22^{\circ} 52^{\prime} 11^{\prime \prime} 87$ & $12^{\mathrm{h}} 53^{\mathrm{m}} 46^{\mathrm{s}} .58$ & $+27^{\circ} 21^{\prime} 06^{\prime \prime} 67$ \\
\hline $02^{\mathrm{h}} 12^{\mathrm{m}} 54^{\mathrm{s}} .59$ & $+06^{\circ} 51^{\prime} 42^{\prime \prime} 78$ & $12^{\mathrm{h}} 53^{\mathrm{m}} 55^{\mathrm{s}} \cdot 05$ & $+62^{\circ} 17^{\prime} 08^{\prime \prime} 94$ \\
\hline $02^{\mathrm{h}} 53^{\mathrm{m}} 02^{\mathrm{s}} \cdot 20$ & $-01^{\circ} 40^{\prime} 19^{\prime \prime} 44$ & $13^{\mathrm{h}} 32^{\mathrm{m}} 01^{\mathrm{s}} \cdot 90$ & $+12^{\circ} 10^{\prime} 17^{\prime \prime} 53$ \\
\hline $08^{\mathrm{h}} 22^{\mathrm{m}} 17^{\mathrm{s}} .55$ & $+37^{\circ} 46^{\prime} 20^{\prime \prime} 67$ & $15^{\mathrm{h}} 38^{\mathrm{m}} 31^{\mathrm{s}} .31$ & $+43^{\circ} 02^{\prime} 01 ! 23$ \\
\hline $09^{\mathrm{h}} 39^{\mathrm{m}} 28^{\mathrm{s}} .81$ & $+22^{\circ} 39^{\prime} 40^{\prime \prime} 76$ & $15^{\mathrm{h}} 41^{\mathrm{m}} 05^{\mathrm{s}} \cdot 11$ & $+09^{\circ} 00^{\prime} 54^{\prime \prime} 75$ \\
\hline $09^{\mathrm{h}} 50^{\mathrm{m}} 13^{\mathrm{s}} .97$ & $+49^{\circ} 39^{\prime} 36^{\prime \prime} 40$ & $15^{\mathrm{h}} 48^{\mathrm{m}} 54^{\mathrm{s}} .99$ & $+62^{\circ} 24^{\prime} 48^{\prime \prime} 04$ \\
\hline $10^{\mathrm{h}} 03^{\mathrm{m}} 39^{\mathrm{s}} \cdot 48$ & $+16^{\circ} 44^{\prime} 55^{\prime \prime} 61$ & $16^{\mathrm{h}} 10^{\mathrm{m}} 01.70$ & $+61^{\circ} 31^{\prime} 06^{\prime \prime} 88$ \\
\hline $10^{\mathrm{h}} 55^{\mathrm{m}} 38^{\mathrm{s}} .50$ & $+14^{\circ} 55^{\prime} 29^{\prime \prime} 15$ & $16^{\mathrm{h}} 24^{\mathrm{m}} 00^{\mathrm{s}} \cdot 44$ & $+61^{\circ} 20^{\prime} 59^{\prime \prime} 43$ \\
\hline $11^{\mathrm{h}} 07^{\mathrm{m}} 51^{\mathrm{s}} .05$ & $+35^{\circ} 02^{\prime} 12^{\prime \prime} 17$ & $16^{\mathrm{h}} 34^{\mathrm{m}} 26^{\mathrm{s}} \cdot 23$ & $+34^{\circ} 04^{\prime} 25^{\prime \prime} 11$ \\
\hline $11^{\mathrm{h}} 24^{\mathrm{m}} 24^{\mathrm{s}} .06$ & $+02^{\circ} 07^{\prime} 26^{\prime \prime} 26$ & $18^{\mathrm{h}} 30^{\mathrm{m}} 56^{\mathrm{s}} \cdot 35$ & $+40^{\circ} 54^{\prime} 17^{\prime \prime} 80$ \\
\hline $11^{\mathrm{h}} 49^{\mathrm{m}} 31^{\mathrm{s}} .87$ & $+16^{\circ} 07^{\prime} 15^{\prime \prime} 09$ & $18^{\mathrm{h}} 53^{\mathrm{m}} 29^{\mathrm{s}} \cdot 27$ & $+77^{\circ} 54^{\prime} 56^{\prime \prime} 64$ \\
\hline
\end{tabular}

Note. For the events listed in Table 2, we remeasured the coordinates of the interesting candidates. The list contains all events showing a single point source with $r<18.4$, either as measured by the listed USNO magnitudes or when we remeasured its magnitude directly from the digitalized plates. Also one object that appears to be seen in more than one image is included.

(as opposed to the radio searches), and earthlings already have the necessary technology to produce short, nanosecond laser pulses that could outshine our own Sun by a factor of $\sim 5000$. SETI programs such as the "Panoramic optical and nearinfrared SETI instrument" (PANOSETI) are currently preparing instrumentation to search for short light pulses on 
timescales of nanoseconds to microseconds that may arise due to interstellar communication (Maire et al. 2018; Wright et al. 2018).

A number of ongoing optical search programs have already succeeded in producing some upper limits to the incidence of both pulsed lasers and continuous laser signals. For example, for $800 \mathrm{~nm}$ lasers, one estimates the upper limit on the fraction of transmitting civilizations to be around $f \sim 10^{-7}$ (NASA Technosignatures Workshop Participants 2018) for $100 \mathrm{~s}$ long pulses. A study that instead looked for signs of a continuous laser in the optical spectra of 5600 FGKM stars (Tellis \& Marcy 2015, 2017) could also exclude the presence of lasers in all of these spectra. Similar searches have been done in the infrared, where extinction is much less of a problem and a wavelength window is opened up that is largely devoid of background noise. See Wright et al. (2014).

The VASCO project may be a "conventional" astrophysics project, but it originated in the context of SETI, as described by Villarroel et al. (2016), who proposed to search surveys for vanished stars in our Galaxy as probes of "impossible effects" that could only be ascribed to an extraterrestrial technology, due to the high likelihood of this as an observational signature. While VASCO attempts to search for more transients of also natural astrophysical origins, the project bears implications for SETI research. A general review describing the possibilities of technosignature searches in time-domain astronomy is given by Davenport (2019).

In the VASCO searches we may search for vanished stars and can expect to find transients on three different timescales: (1) a hypothetical vanished star may have existed for billions of years before it vanishes. We have determined that the probability is $p<10^{-7}$. (2) We may find extreme, variable astrophysical objects that vary over timescales of decades. (3) We may find astrophysical transients that are as short as the exposure time of a typical POSS image $(\sim 1 \mathrm{hr})$. The short transient detections we see in the red plates from POSS-I may have many different explanations, ranging from instrumental causes to bona fide astrophysical ones. An attractive feature about the list we have produced is that a monochromatic interstellar laser at $600-680 \mathrm{~nm}$ that shines for about one hour may well present itself as a point source detected only once in one image, due to the short time when the laser operated. Simply put, the single events presented in Section 3.3 have many degenerate solutions. It will be the work of a future publication to work out and disentangle this.

In SETI, frequent technosignature searches also include searches for giant structures that harness the energy of stars and produce waste heat with temperatures $T \sim 100-300 \mathrm{~K}$. The most extreme form is referred to as a Dyson sphere (Dyson 1960), which entirely encloses a star and produces the largest fractional change in the brightness of the object. Carrigan (2009) sought Dyson spheres around 11,000 stars using IRAS photometry and spectroscopy. Zackrisson et al. (2015) surveyed 1359 galaxies with the help of the Tully-Fischer relation, found no convincing candidates, and estimated that the fraction of Kardashev II-III civilisations (Kardashev 1964) capable of transforming their entire galaxy is less than $0.3 \%$. Griffith et al. (2015) used WISE and the Two Micron All Sky Survey to search for IR excesses among 100,000 targets that appear to be dust-rich, star-forming galaxies. As the waste heat shows the same signatures that dust shows, for extragalactic objects these searches may be too ambiguous to give confirmable candidates.

One may wonder why a highly advanced Kardashev II-III civilisation, capable of putting Dyson spheres around every star in a galaxy, would limit their effort to harness the energy of stars over such a giant volume as an entire galaxy. Indeed, an AGN occupies a much smaller space (as small as our solar system), and has much more concentrated energy to offer. For example, the quasar 3C 273 has about 4 trillion times the luminosity of our Sun. Indeed, an AGN may be a significantly more effective target to build a Dyson sphere around. Many AGNs (in particular obscured ones) naturally have a thick layer of dust dimming the central power source and emitting infrared radiation. This dust is located at the sublimation radius. When an AGN is so obscured that hardly any photons leak through to excite the surrounding gas, we may not even detect the typical narrow emission lines that are the signature of an AGN.

When the accretion disk varies and changes its intensity, we expect the corresponding hot dust emission (arising typically $\sim 0.1 \mathrm{pc}$ from the supermassive black hole) to respond, but with a time delay. This time delay is often used to infer the physical size of the black hole. Together with the angular size of the torus, obtainable from interferometry, one can estimate the distance to the AGN using it as a standard candle (Hoenig et al. 2014).

However, in a dynamic, Dysonian AGN one may expect that the time delay of the infrared emission does not follow the typical behavior of a dust torus. It could be that the AGN cannot even be used as a standard candle, because the artificial structure will not obey natural changes in the power source. Therefore, as an extension of the VASCO project, we suggest searches for extragalactic objects with variability in the infrared region. These variable AGNs can be followed up with IR reverberation mapping experiments. The research will mainly be aimed at understanding the mysterious nature of the central few parsecs of an AGN.

\subsection{Future Work}

\subsubsection{Giving Extraterrestrials a Second Chance}

Undoubtedly, VASCO will generate large lists of candidate objects in searches for vanishing stars. Individually, these serve no purpose unless verified. We can agree that a wide-field search that results in a list of candidates is of no great interest for research if each candidate sooner or later gets dismissed due to a lack of verification as a potential SETI candidate.

However, if a region of the sky has a tendency to produce an unexpectedly large fraction of candidates relative to the background, this region or "hot spot" may deserve some extra attention. As a part of VASCO's research program, we plan to combine all the unverified initial results from many different search programs, such as the optical all-sky surveys NIROSETI and PANOSETI, and from other wide-field surveys in general (see Section 5.2). We aim to visualize the background of the unverified candidates in a two-dimensional projection of the sky. Altogether, this noisy background of neglected candidates could reveal "hot spots" of transient activity, where for some reason many candidates are concentrated. Doing this iteratively with reliable clustering methods and zooming in on the most active regions in our SETI (or technosignature) searches, we can identify the most probable locations to host extraterrestrial intelligence. VASCO will therefore never dismiss any 
candidates forever. Rejection and acceptance are only transient states in the process. The information on potential "hot spots" can further be used to select the most interesting candidates.

\subsubsection{Expanding the Set of Candidates}

What we have presented so far is a cross-match between USNO and Pan-STARRS in searches for vanishing objects, using a $30^{\prime \prime}$ cross-match radius. However, the plan of VASCO is to do the following:

1. Finalize the current search for vanishing objects with a $30^{\prime \prime}$ cross-match radius by examining the entire Mismatch Sample visually and finalize the cross-match over the sky regions that so far have not been used.

2. Search for appearing objects within a 30" cross-match radius (with Pan-STARRS objects having $r<19$ ).

3. Search for vanishing objects within a $5^{\prime \prime}$ cross-match radius (setting limits in magnitude), including corrections for proper motion. Given the larger number of spurious mismatches with this search radius, we will need to develop better automatic methods to handle the identification of candidates in images.

This is a long-winded process requiring considerable time on powerful computing clusters, but it may generate a large list of interesting transients of all sorts.

The large number of images we are dealing with within the complete VASCO project and the increased complexity of our searches require a better approach than was followed in the pilot study. Clearly, we must explore ways to avail ourselves of automated procedures as much as possible, but without relying on algorithms for all candidate selection and quality control. At this moment, such algorithms are still being developed. Current problems are related to the inefficiency of comparing two images manually and comparing images based on CCD cameras with images from old photographic plates; finally we must adjust the algorithms to identify the most meaningful candidates. In a separate paper by K. Pelckmans et al. (2019, in preparation). we propose a new tool for handling a large number of images using methods of machine learning.

\subsection{Summary}

VASCO is a project that provides an opportunity to discover many past transients events, both objects that vanish and those that appear. The time span between these surveys is large. This allows for phenomena to be discovered other than what can be expected in ongoing transient surveys such as, e.g., ZTF. Using a large cross-match radius of $30^{\prime \prime}$, we obtained a sample of 150,000 USNO objects that cannot to be found in PanSTARRS. This represents an interesting starting sample in searches for vanishing objects. As we used a large cross-match radius of $30^{\prime \prime}$ (instead of the more typical $3^{\prime \prime}-5^{\prime \prime}$ ), we underestimate the real number of potential mismatches that can be found through cross-matching attempts. We have investigated the statistical properties of this sample and found that many of these "mismatches" are occurring in the red band. Visual checks confirm that indeed the most interesting cases, about 100, are mostly one-time detections in the red band. At present, we do not know what these detections represent. We believe they may be a mixed bag of transient phenomena. The object found by Villarroel et al. (2016) is of the same class, and might possibly be a variable object that dropped 4.5 mag since it was imaged long ago. It could also have been some type of transient event such as a background high-redshift supernova or a flaring $\mathrm{M}$ dwarf.

In good agreement with theoretical predictions for the number of failed supernovae in our Galaxy (see Appendix), we also set an upper limit on the probability of detecting a vanishing star to be less than 1 in 90 million during our time window of $70 \mathrm{yr}$.

Meanwhile, we will keep developing methods to analyze the remaining images in the Mismatch Sample in searches for reliable examples of vanishing stars.

Based on observations made with the Nordic Optical Telescope operated on the island of La Palma by NOTSA in the Spanish Observatorio del Roque de los Muchachos of the Instituto de Astrofísica de Canarias under the joint IACNordic Observing Time programme. This article is also based on observations made with the IAC80 telescope operated on the island of Tenerife by the Instituto de Astrofisica de Canarias in the Spanish Observatorio del Teide.

The cross-match computations were performed on resources provided by the Swedish National Infrastructure for Computing (SNIC) at UPPMAX. Salman Toor assisted in operating the resources.

B.V. wishes to thank Geoffrey Marcy for many ideas, discussions, and suggestions that improved the project and the manuscript. B.V. wishes to thank Anders Nyholm for good discussions, and also thank Kjell Lundgren, Lars Hermansson, Abel Souza, and Jason Wright. Particular thanks to Nidia Morell who helped with the Magellan images.

B.V. is funded by the Swedish Research Council (Vetenskapsrådet, grant No. 2017-06372). B.V. also wishes to thank the Department of information technology at Uppsala University for supporting this project. B.V. wishes further to thank Bernie Shiao from the Pan-STARRS collaboration for the offline Pan-STARRS DR1 data set on which all VASCO work is built.

The Pan-STARRS1 Surveys (PS1) and the PS1 public science archive have been made possible through contributions by the Institute for Astronomy, the University of Hawaii, the Pan-STARRS Project Office, the Max-Planck Society and its participating institutes, the Max Planck Institute for Astronomy, Heidelberg and the Max Planck Institute for Extraterrestrial Physics, Garching, The Johns Hopkins University, Durham University, the University of Edinburgh, Queen's University Belfast, the Harvard-Smithsonian Center for Astrophysics, the Las Cumbres Observatory Global Telescope Network Incorporated, the National Central University of Taiwan, the Space Telescope Science Institute, the National Aeronautics and Space Administration under grant No. NNX08AR22G issued through the Planetary Science Division of the NASA Science Mission Directorate, National Science Foundation grant No. AST-1238877, the University of Maryland, Eotvos Lorand University (ELTE), the Los Alamos National Laboratory, and the Gordon and Betty Moore Foundation.

Funding for the Sloan Digital Sky Survey IV has been provided by the Alfred P. Sloan Foundation, the U.S. Department of Energy Office of Science, and the Participating Institutions. SDSS-IV acknowledges support and resources from the Center for High-Performance Computing at the University of Utah. The SDSS website is www.sdss.org. 
SDSS-IV is managed by the Astrophysical Research Consortium for the Participating Institutions of the SDSS Collaboration including the Brazilian Participation Group, the Carnegie Institution for Science, Carnegie Mellon University, the Chilean Participation Group, the French Participation Group, Harvard-Smithsonian Center for Astrophysics, Instituto de Astrofísica de Canarias, The Johns Hopkins University, Kavli Institute for the Physics and Mathematics of the Universe (IPMU)/University of Tokyo, the Korean Participation Group, Lawrence Berkeley National Laboratory, Leibniz Institut für Astrophysik Potsdam (AIP), Max-Planck-Institut für Astronomie (MPIA Heidelberg), Max-Planck-Institut für Astrophysik (MPA Garching), Max-Planck-Institut für Extraterrestrische Physik (MPE), National Astronomical Observatories of China, New Mexico State University, New York University, University of Notre Dame, Observatário Nacional/MCTI, The Ohio State University, Pennsylvania State University, Shanghai Astronomical Observatory, United Kingdom Participation Group, Universidad Nacional Autónoma de México, University of Arizona, University of Colorado Boulder, University of Oxford, University of Portsmouth, University of Utah, University of Virginia, University of Washington, University of Wisconsin, Vanderbilt University, and Yale University.

\section{Appendix \\ Estimated Failed Supernova Rates}

Failed supernovae (SNe) happen when massive stars do not explode as $\mathrm{SNe}$, but rather collapse into black holes without any preceding transient event (see, e.g., Kochanek et al. 2008; Smartt et al. 2009; Ugliano et al. 2012; Adams et al. 2016, 2017a, 2017b; Ertl et al. 2016). The concept is mainly theoretical, because no failed SN has been found observationally with absolute certainty, although there exists at least one good candidate (Adams et al. 2016). Despite the scarcity of empirical evidence, there is still reason to believe that there is a deficit of higher mass $\mathrm{SN}$ progenitors as identified by Kochanek et al. (2008). But how likely is it that such a failed SN even occurs in the Galaxy within the VASCO timeframe?

To assess the probability of detecting a failed $\mathrm{SN}$ we need to consider two things: (1) the formation rate of massive stars in the Galaxy and (2) which stars (i.e., stars of what mass range) are the progenitors of the failed $\mathrm{SNe}$. The first can be calculated from the total star formation rate for a given stellar initial mass function (IMF). The latter can be estimated from theoretical SN models, but unfortunately these models do not agree very well regarding complete fallback and direct black hole formation (see, e.g., O’Connor \& Ott 2011, for further details).

Most observational estimates of the total Galactic star formation rate (SFR) made over the last four decades fall in the range $0.5-8 M_{\odot} \mathrm{yr}^{-1}$, depending on assumptions about the IMF (see, e.g., Diehl et al. 2006; Robitaille \& Whitney 2010, and references therein). Here, we will use the value of Diehl et al. (2006), namely $\dot{M}_{\mathrm{SFR}}=3.8 M_{\odot} \mathrm{yr}^{-1}$, which is almost exactly in the middle of the above range. This is based on the IMF of Scalo (1986), which has a power-law tail at the highmass end with slope -2.7 . In the following, we shall treat as more or less free parameters the high-mass slope, the fraction $f_{\text {LIMS }}$ of low- and intermediate-mass stars (LIMS), and the fraction $f_{\mathrm{HMS}}$ of high-mass stars (HMS). We will use the fractions $f_{\text {LIMS }}$ and $f_{\text {HMS }}=1-f_{\text {LIMS }}$ interchangeably.

On the $\sim 100$ yr timescale of the VASCO project it is fair to assume a constant Galactic-average SFR. Thus, the expected
$\mathrm{SN}$ rate $\mathcal{R}_{\mathrm{SN}}$ is given by

$$
\mathcal{R}_{\mathrm{SN}}=\dot{M}_{\mathrm{SFR}} \times \int_{m_{\mathrm{SN}}}^{m_{\max }} \phi(m) d m
$$

with the IMF expressed as

$$
\phi\left(m, m_{0}, \epsilon\right)=\phi_{0}\left(\frac{m}{m_{0}}\right)^{-(1+\epsilon)} f_{\text {LIMS }}\left(m_{0}, m_{\mathrm{SN}}, \epsilon\right),
$$

where $\phi_{0}$ is a normalization constant chosen such that the average mass $\langle m\rangle=1, m_{\mathrm{SN}}$ is the lower mass limit for $\mathrm{SNe}, m_{0}$ is the turnover mass, i.e., the stellar mass where the IMF peaks and then turns over, and $\epsilon$ is the logarithmic slope of the highmass end. The LIMS fraction $f_{\text {LIMS }}$ is then also a function such that

$$
f_{\mathrm{LIMS}}=\int_{m_{\min }}^{m_{\mathrm{SN}}} \phi\left(m, m_{0}, \epsilon\right) d m / \int_{m_{\min }}^{m_{\max }} \phi\left(m, m_{0}, \epsilon\right) d m .
$$

The canonical IMF of Salpeter (1955) corresponds to $\epsilon=1.35$ and $f_{\mathrm{HMS}}=0.003$. To calculate the rate of failed $\mathrm{SNe}$, we simply need to replace the $\mathrm{SN}$ mass domain $\mathcal{M}_{\mathrm{SN}} \equiv$ $\left[m_{\mathrm{SN}}, m_{\mathrm{max}}\right]$ with the corresponding mass domain for failed $\mathrm{SNe}, \mathcal{M}_{\mathrm{FSN}}$. We consider two estimates of $\mathcal{M}_{\mathrm{FSN}}$, based on the results of Limongi \& Chieffi (2006, henceforth LC06) and Woosley \& Heger (2007, henceforth WH07), which were chosen because the fraction of failed $\mathrm{SNe}$ in these studies is high enough (also at solar metallicity) to have a notable effect on the SN rate (see also O'Connor \& Ott 2011).

Based upon the simple SN-rate model above, we have explored how the rate of failed SNe depends on $\epsilon$ and $f_{\text {LIMS }}$ or $f_{\mathrm{HMS}}$ for the two estimates of $\mathcal{M}_{\mathrm{FSN}}$. The results are shown in Figure 13, from which it is clear that only a top-heavy IMF can produce a rate high enough for it to be likely that we find failed SNe within VASCO. For both the LC06 and the WH07 cases an IMF slope $\epsilon=1.35$ indicates that no failed SN should be detected over a $50 \mathrm{yr}$ timescale $\left(\mathcal{R}_{\mathrm{FSN}}<0.005 \mathrm{yr}^{-1}\right)$, for all reasonable values of $f_{\text {LIMS }}$ (or $f_{\mathrm{HMS}}$ ). In order to reach two $\mathrm{SNe}$ per century, or above, we have to invoke an extremely topheavy present-day IMF. Essentially, all recent observational constraints on the Galactic IMF indicate an IMF that is actually steeper than the Salpeter IMF $(\epsilon>1.35)$ and with no strong evidence for variation (see Bastian et al. 2010, and references therein), suggesting that it is unlikely that we would catch a failed SN in the Galaxy, although it cannot be ruled out.

Our choice of the LC06 and WH07 results as a basis for calculating the rate of failed $\mathrm{SNe}$ is somewhat arbitrary, but also conservative. It must be emphasized that it is not well known how common failed $\mathrm{SNe}$ actually are. A mass range [18 $\left.M_{\odot}, 25 M_{\odot}\right]$ is sometimes quoted (e.g., Smartt et al. 2009; Adams et al. 2017a), and theoretical work does indeed seem to lend some support to it (O'Connor \& Ott 2011; Ugliano et al. 2012; Ertl et al. 2016). For a normal IMF $(\epsilon=1.35)$, $\mathcal{M}_{\mathrm{FSN}} \equiv\left[18 M_{\odot}, 25 M_{\odot}\right]$ leads to a rate $\mathcal{R}_{\mathrm{FSN}} \sim 0.025 \mathrm{yr}^{-1}$. In such a case, the prospect of finding a failed $\mathrm{SN}$ within a $50 \mathrm{yr}$ window is somewhat better. Assuming that no stars in the initial-mass range $\left[18 M_{\odot}, 25 M_{\odot}\right]$ will explode is perhaps too extreme.

In summary, "failed SNe"-massive stars that collapse to black holes without any detectable transient event (SN explosion)_are not likely to explain vanishing stars in the 

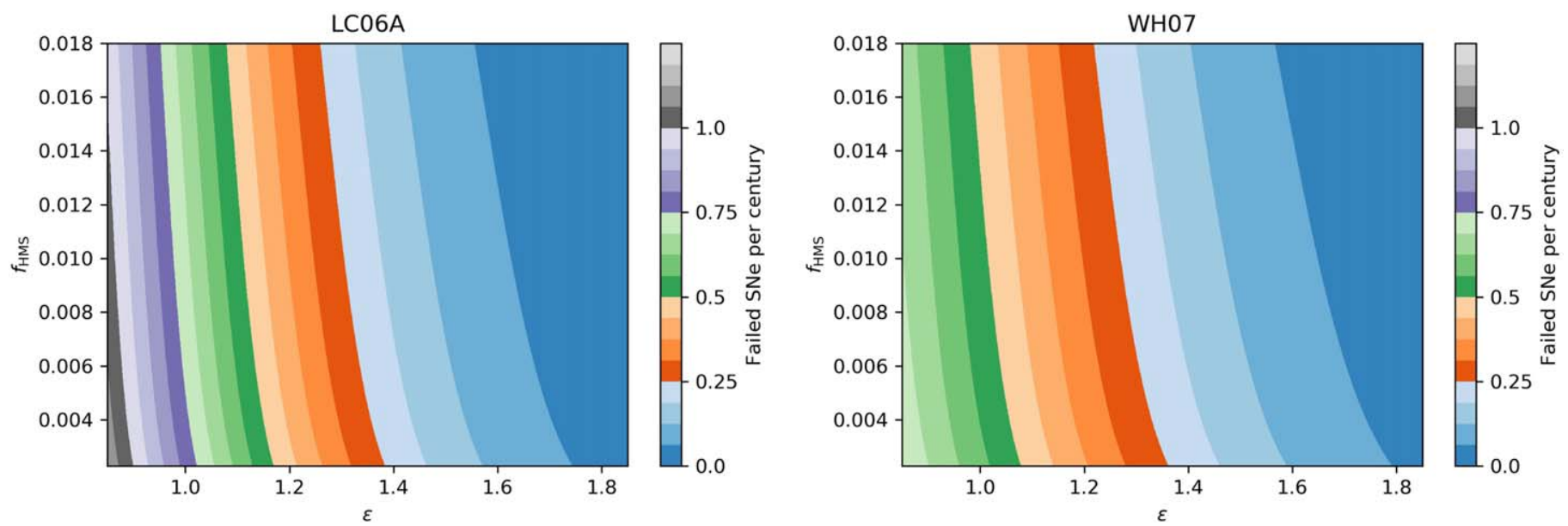

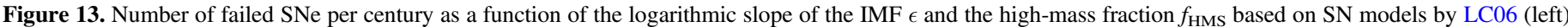
and WH07 (right).

Galaxy on timescales less than $\sim 1000$ yr. Our current understanding of the progenitors of failed $\mathrm{SNe}$ (such as the models of LC06 and WH07) indicates that such events should be caught by VASCO only if the present-day IMF is extremely top-heavy. On a more speculative note, failed $\mathrm{SNe}$ are not well understood. Therefore, we cannot rule out the possibility that less massive (and therefore more abundant) stars fail to explode, possibly due to some other mechanism, thus leading to a much higher rate of "vanishing stars."

\section{ORCID iDs}

Martín López-Corredoira (iD https://orcid.org/0000-00016128-6274

Kevin Krisciunas (iD https://orcid.org/0000-0002-6650-694X Eduardo Guerras (ib) https://orcid.org/0000-0001-5534-4458 Oleg Kochukhov (iD https://orcid.org/0000-0003-3061-4591 J. Emilio Enriquez (ib https://orcid.org/0000-0003-2516-3546 Alok C. Gupta (1D https://orcid.org/0000-0002-9331-4388

\section{References}

Adams, S. M., Kochanek, C. S., Gerke, J. R., \& Stanek, K. Z. 2017a, MNRAS, 469, 1445

Adams, S. M., Kochanek, C. S., Gerke, J. R., Stanek, K. Z., \& Dai, X. 2017b, MNRAS, 468, 4968

Adams, S. M., Kochanek, C. S., Prieto, J. L., et al. 2016, MNRAS, 460, 1645

Barron, J. T., Stumm, C., Hogg, D. W., Lang, D., \& Roweis, S. 2008, ApJ, 135,414

Bastian, N., Covey, K. R., \& Meyer, M. R. 2010, ARA\&A, 48, 339

Bond, H. E., Bedin, L. R., Bonanos, A. Z., et al. 2009, ApJL, 695, L154

Carrigan, R. A. 2009, ApJ, 698, 2075

Chambers, K. C., Magnier, E. A., Metcalfe, N., et al. 2016, arXiv:1612.05560

Clayton, G. C. 2012, JAVSO, 40, 539

Davenport, J. 2019, arXiv:1907.04443

Diehl, R., Halloin, H., Kretschmer, K., et al. 2006, Natur, 439, 45

Djorgovski, S. G. 2000, in ASP Conf. Ser. 213, A New Era in Bioastronomy, ed. G. Lemarchand \& K. Meech (San Francisco, CA: ASP), 519

Djorgovski, S. G., Gal, R. R., Odewahn, S. C., et al. 1998, BAAS, 30, 1270

Djorgovski, S. G., Mahabal, A. A., Brunner, R. J., et al. 2001, in ASP Conf. Ser. 225, Virtual Observatories of the Future, ed. R. Brunner, S. G. Djorgovski, \& A. Szalay (San Francisco, CA: ASP), 52

Djorgovski, S. G., Drake, A. J., Mahabal, A. A., et al. 2012, arXiv:1102.5004 Drake, A. J., Djorgovski, S. G., Graham, M. J., et al. 2019, MNRAS, 482, 98 Drake, A. J., Djorgovski, S. G., Mahabal, A., et al. 2009, ApJ, 696, 870 Dyson, F. 1960, Sci, 131, 1667

Ertl, T., Janka, H.-T., Woosley, S. E., Sukhbold, T., \& Ugliano, M. 2016, ApJ, 818,124
Flewelling, H. A., Magnier, E. A., Chambers, K. C., et al. 2016, arXiv:1612. 05243

Gaia Collaboration, Brown, A. G. A., Vallenari, A., et al. 2018a, A\&A, 595, A1

Gaia Collaboration, Katz, D., Antoja, T., et al. 2018b, A\&A, 616, A11

Graham, M. J., Djorgovski, S. G., Drake, A. J., et al. 2017, MNRAS, 470, 4112

Graham, M. J., Ross, N. P., Stern, D., et al. 2019, arXiv:1905.02262

Greiner, J., Wenzel, W., \& Degel, J. 1990, A\&A, 234, 251

Griffith, R. L., Wright, J. T., Maldonado, J., et al. 2015, ApJS, 217, 25

Grindlay, J., Tang, S., Los, E., \& Servillat, M. 2012, in Proc. IAU Symp. 285 , New Horizons in Time-Domain Astronomy, ed. E. Griffin, R. Hanisch, \& R. Seaman (Cambridge: Cambridge Univ. Press), 29

Hippke, M., Learned, J. G., Zee, A., et al. 2015, ApJ, 798, 42

Hoenig, S. F., Watson, D., Kishimoto, M., \& Hjorth, J. 2014, Natur, 515, 528

Kaiser, N., Aussel, H., Burke, B. E., et al. 2002, Proc. SPIE, 4836, 154

Kankare, E., Kotak, R., Mattila, S., et al. 2017, NatAs, 1, 865

Kardashev, N. S. 1964, SvA, 8, 217

Kochanek, C. S., Beacom, J. F., Kistler, M. D., et al. 2008, ApJ, 684, 1336

Lawrence, A., Bruce, A. G., MacLeod, C., et al. 2016, MNRAS, 463, 296

Limongi, M., \& Chieffi, A. 2006, ApJ, 647, 483

Mack, K. H., Prieto, M. A., Brunetti, G., \& Orienti, M. 2009, MNRAS, 392, 705

Madsen, G. J., \& Gaensler, B. M. 2013, ApJS, 209, 33

Magnier, E. A., Chambers, K. C., Flewelling, H. A., et al. 2016a, arXiv:1612. 05240

Magnier, E. A., Schlafly, E. F., Finkbeiner, D. P., et al. 2016b, arXiv:1612. 05242

Magnier, E. A., Sweeney, W. E., Chambers, K. C., et al. 2016c, arXiv:1612. 05244

Mahabal, A. A., Djorgovski, S. G., Drake, A. J., et al. 2009, ATel, 2051, 1

Mahabal, A. A., Djorgovski, S. G., Drake, A. J., et al. 2011, BASI, 39, 387

Maire, J., Wright, S. A., Cosens, M., et al. 2018, Proc. SPIE, 10702, 107025L

Mattsson, L., \& Villarroel, B. 2017, Populär Astronomi, 3, 18

Monet, D. G., Levine, S. E., Canzian, B., et al. 2003, ApJ, 125, 984

NASA Technosignatures Workshop Participants 2018, arXiv:1812.08681

O’Connor, E., \& Ott, C. D. 2011, ApJ, 730, 70

Prieto, M. A. 1997, MNRAS, 284, 627

Robitaille, T. P., \& Whitney, B. A. 2010, ApJL, 710, L11

Rodriguez, R., Schmidt, S. J., Jayasinghe, T., et al. 2018, RNAAS, 2, 2

Salpeter, E. E. 1955, ApJ, 121, 161

Samus, N. N., Kazarovets, E. V., Durlevich, O. V., Kireeva, N. N., \& Pastukhova, E. N. 2017, ARep, 61, 80

Scalo, J. M. 1986, FCPh, 11, 1

Schwartz, R. N., \& Townes, C. H. 1961, Natur, 190, 205

Smartt, S., Eldridge, J. J., Crockett, R. M., \& Maund, J. R. 2009, MNRAS, 395, 1409

Soodla, J. 2019, A System for Cross-matching All-sky Surveys, Master thesis, Uppsala Universitet

Tang, S., Grindlay, J., Los, E., \& Laycock, S. 2010, ApJL, 710, L77

Tang, S., Grindlay, J., Moe, M., et al. 2012, ApJ, 751, 99

Tellis, N. K., \& Marcy, G. W 2015, PASP, 127, 450

Tellis, N. K, \& Marcy, G. W 2017, ApJ, 153, 251

Tisserand, P., Clayton, G. C., Bessell, M. S., et al. 2018, arXiv:1809.01743 
Ugliano, M., Janka, H.-T., Marek, A., \& Arcones, A. 2012, ApJ, 757, 69 Vavilova, I. B. 2016, OAP, 29, 109

Vavilova, I. B., Pakulyak, L. K., Shlyapnikov, A. A., et al. 2012, KPCB, 28, 85

Villarroel, B., Imaz, I., \& Bergstedt, J. 2016, AJ, 152, 76

Waters, C. Z., Magnier, E. A., Price, P. A., et al. 2016, arXiv:1612.05245

Woosley, S. E., \& Heger, A. 2007, PhR, 442, 269
Wright, S. A., Horowitz, P., Maire, J., et al. 2018, Proc. SPIE, 10702, 107025I Wright, S. A., Werthimer, D., Treffers, R. R., et al. 2014, Proc. SPIE, 9147, 91470J

York, D. G., Adelman, J., Anderson, J. E., et al. 2000, AJ, 120, 1579

Zackrisson, E., Calissendorff, P., Asadi, S., \& Nyholm, A. 2015, ApJ, 810, 